\title{
Fully isogeometric modeling and analysis of nonlinear 3D beams with spatially varying geometric and material parameters
}

\author{
Oliver Weeger $^{\mathrm{a}, *}$, Sai-Kit Yeung ${ }^{\mathrm{b}}$, Martin L. Dunn ${ }^{\mathrm{c}}$ \\ ${ }^{a}$ Singapore University of Technology and Design, Information Systems Technology and Design Pillar \\ 85 SUTD Digital Manufacturing and Design Centre, 8 Somapah Road, Singapore 487372, Singapore \\ ${ }^{b}$ Hong Kong University of Science and Technology, Division of Integrative Systems and Design, \\ Room 6530, 6/F, School of Engineering, Clear Water Bay, Kowloon, Hong Kong \\ ${ }^{c}$ University of Colorado Denver, College of Engineering and Applied Science, \\ Campus Box 104, Denver, CO 80217-3364, United States
}

\begin{abstract}
We present a fully isogeometric modeling and simulation method for geometrically exact, nonlinear 3D beams with spatially varying geometric and material distributions, both along the beam axis and through its cross-section. The approach is based on the modeling of 3D beams using the Cosserat rod theory and the numerical discretization using B-Spline and NURBS parameterizations in an isogeometric collocation method. Transversally varying material constitutions are represented using non-homogeneous, functionally graded beam cross-section definitions such as laminates and continuously graded cross-sections. Furthermore, to model the axial variation of material and geometry, we introduce the parameterization of cross-section properties as spline curves along the beam centerlines. This fully isogeometric modeling and analysis concept, which is based on spline parameterizations of initial beam centerline curves, kinematic unknowns and axially varying material and geometric parameters, has various practical applications enabled by advances in manufacturing technology, including multi-material 3D printing and advanced manufacturing of composites with automated fiber placement. We verify and demonstrate the modeling and simulation approach using several numerical studies and highlight its practical applicability.
\end{abstract}

Keywords: Isogeometric analysis, 3D beams, Spatially varying materials, Functionally graded beams, Collocation method

\section{Introduction}

In recent years, many new possibilities for design and manufacturing of slender and light-weight structures have emerged through the advancement of additive manufacturing technologies [1. Existing and potential applications range from three-dimensionally (3D) printed micro-structures, which can be used to create meta-materials with high strength-to-weight ratios [2, 3, to multi-functional and composite materials with locally defined material properties [4-6] (see Fig. 1a), to active, smart and self-assembling materials and structures, soft robots, and deployable composite space structures, where compliant components need to be designed with tailored large deformation behavior [7-12] (see Fig. 1b). In particular, multi-method and multi-material 3D printing now enable the fabrication of freeform structures with arbitrarily varying material compositions, thus opening new perspectives for design and application of spatially varying and composite materials.

Modeling and design of these structures, especially when they are made from soft materials and subject to large deformations, calls for advanced simulation methods and computer-aided engineering tools that can

\footnotetext{
${ }^{*}$ Corresponding author

Email addresses: oliver_weeger@sutd.edu.sg (Oliver Weeger), saikit@ust.hk (Sai-Kit Yeung), martin.dunn@ucdenver.edu (Martin L. Dunn)
} 


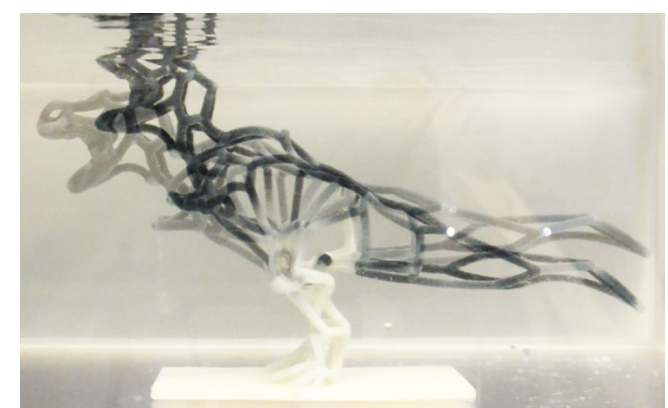

(a) Active shape memory polymer structure with axially varying Young's modulus distribution [6]

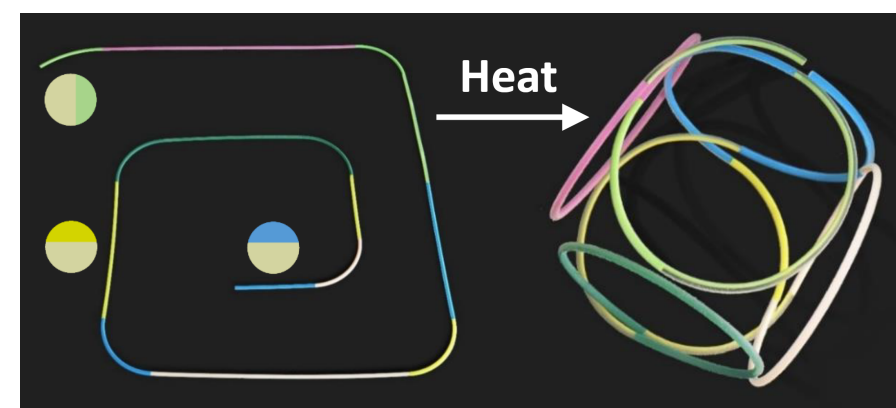

(b) Direct 4D printed structure with transversally varying laminate cross-section with axially varying layer orientation [10]

Figure 1: Examples of 3D beam structures with spatially varying materials and geometries (both axially varying and transversally varying) undergoing large deformations and fabricated through additive manufacturing, here multi-material 3D printing

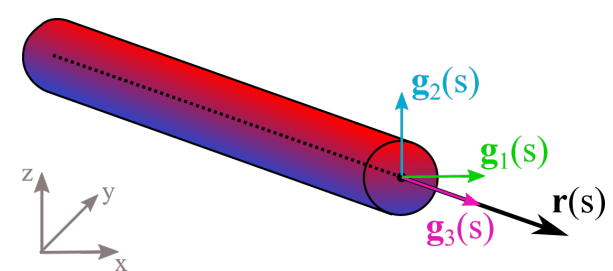

(a) Rod with transversally varying (TV) material distribution, illustrated by color change from red to blue through the cross-section

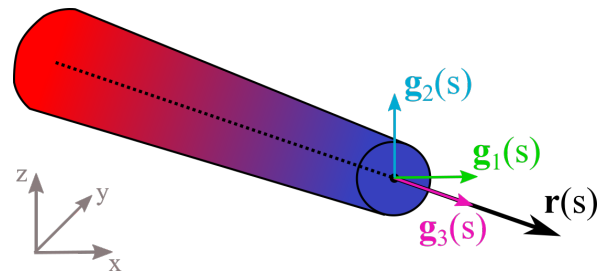

(b) Rod with axially varying (AV) material distribution and radius, illustrated by color change along the centerline from red to blue and decreasing cross-section radius from back to front

Figure 2: Configuration of the Cosserat rod as a framed curve with centerline $\mathbf{r}(s)$ and orthonormal frames $\mathbf{R}(s)=$ $\left(\mathbf{g}_{1}(s), \mathbf{g}_{2}(s), \mathbf{g}_{3}(s)\right)$ defining the cross-section orientations. Colors illustrate the spatial variation of material properties of a circular cross-section (a) with transversally varying material through the cross-section and(b) axially varying material and radius along the centerline

efficiently incorporate the complex material constituency. Thus, there is a demand for nonlinear modeling and simulation of 3-dimensional beam structures with spatially varying material properties. In the context of this work, we distinguish two types of spatial variations: We consider a beam as transversally varying (TV) when its material properties vary through the cross-section of the beam. This type of variation is often called functionally graded in beam literature [13, 14, and can, for instance, be realized through discrete stacking of materials or composites with varying fiber orientations in multiple layers, or continuous grading of material distributions (see Fig. 2a for illustration). Furthermore, an axially varying (AV) beam is characterized by varying parameters of the (homogeneous or transversally varying) cross-section along the centerline of the beam, which could be continuously changing material properties such as Young's modulus or geometric parameters such as radius (see Fig. 2b for illustration).

For modeling and simulation of transversally varying, functionally graded 2D beams, several methods based on Euler-Bernoulli and Timoshenko beam theories [15/17] have been proposed and a beam finite element for functionally graded, i.e., rectangular, layered, beams was introduced including thermoelastic effects 18. Many attempts to model and simulate 3D beams undergoing large elastic deformations are based on the geometrically exact 3D beam theory, usually referred to as Cosserat rod [19, 20, Reissner [21] or Simo 22] beam theory, which is based on the kinematic description of a beam using its centerline position and the orientation of the rigid cross-section. For modeling of transversally varying, functionally graded and composite cross-sections, several extensions and modifications of the theory have been made so far [14]. A geometrically exact formulation for multi-layer beams was presented in [23, where moments and rotations can be discontinuous across layers, thus allowing for the overall beam cross-section to deform. In [24] a variational iteration method was proposed for large deflection analysis of planar curved beams made of functionally graded materials. This work is closely related to [25, 26], where constitutive coefficients of functionally graded 3D beams with general cross-section shapes and composite layouts were derived 
and deformation analysis carried out analytically and via finite element methods (FEM). Further works include the study of nonlinear free vibrations of functionally graded carbon nanotube-reinforced composite beams [27, as well as Timoshenko-like modeling of initially curved and twisted composite beams [28]. The extension of geometrically exact beams to finite strain 3D material models was presented in 29.

For modeling and analysis of beams with axially varying material and cross-section properties, such as tapered, non-prismatic beams, it has been shown that the shear stress distribution depends on all internal forces and moments, which means that it is not exactly reproduced by common Euler-Bernoulli- and Timoshenko-type beam models [30, 31]. To address this issue, for instance, Murín and co-workers have presented finite element formulations for linear analysis of 3D beams with varying cross-section radii [32], nonlinear 3D truss elements with varying stiffness [33] and a 2D beam finite element for modeling of functionally graded beams with spatially varying properties, including electro-thermal-structural multi-physics analysis [34. More recently, a simple Timoshenko-like model for non-prismatic, planar beams was presented [35].

Many different methods have so far been proposed for the numerical discretization of the geometrically exact 3D model and its extension to functionally graded cross-sections, such as finite element methods 3638, finite difference methods 39, 40, and isogeometric collocation methods 41, 42, the latter being the starting point for this work. Isogeometric analysis (IGA) was first proposed in 2005 [43] and has since gained significant popularity in practically all fields of computational mechanics, especially in structural mechanics where many new formulations also for beam models have been proposed [44 47. More recently, isogeometric collocation methods have been introduced [48, 49] as an efficient alternative to finite element methods [50] and were successfully applied to beam models [51 -53], including the Timoshenko-like non-prismatic beam model [54, as well as other types of problems in structural and continuum mechanics, such as elastostatics and dynamics [55], Reissner-Mindlin plates [56], Kirchhoff-Love shells [57], and large deformation elasticity with contact [58].

The basis of isogeometric methods is the parameterization of geometry and discretization of unknowns using B-Splines, NURBS and other spline-type functions, which are commonly used in computer-aided design (CAD). Here, we not only apply an isogeometric discretization, but extend the concept of spline parameterizations to the modeling of axially varying cross-section properties, i.e., material and geometric parameters that change along the centerline of the beam. We demonstrate this fully isogeometric modeling and simulation approach using an isogeometric collocation method for the Cosserat rod model, but the concept could be easily extended to other beam models or discretization methods such as finite elements. Being applied to the geometrically exact 3D beam model, our approach does not consider the above-mentioned dependence of the shear stress distribution on all internal forces and moments for axially varying cross-section properties. Up to the authors' knowledge, such a formulation has not yet been developed in the context of nonlinear, geometrically exact 3D beams and according to 30. this dependence can be neglected if the tapering or, in general, the axial variation of parameters, is small. To validate this assumption, we have carried out comparisons with the planar beam, small deformation applications investigated in [54], where good qualitative and quantitative agreement could be observed with deviations of displacements, forces and moments being not more than $4 \%$. Though this limits the applicability of our fully isogeometric modeling and simulation approach to structures with small axial variation and shear strains, e.g., slender rods, it is still beyond the capabilities of existing tools for the design of complex structures that can be fabricated through advanced, additive manufacturing technologies.

The outline of this manuscript is as follows: In Section 2 we describe the geometrically exact Cosserat rod model and then outline its numerical discretization using the isogeometric collocation method in Sect. 3 . The modeling and implementation of beams with transversally varying materials and functionally graded crosssections in terms of their constitutive coefficients is presented in Sect. 4 and subsequently the isogeometric parameterization of axially varying cross-section properties is introduced. In Section 5 a new mixed method is presented, which resolves shear locking and convergence problems for thin rods with transversally varying cross-sections. The accuracy and convergence behavior of the numerical methods are investigated and confirmed in Sect. 6, and further applications of the modeling and simulation approach with rods and rods structures with TV and AV material and geometric parameters are presented. The paper concludes with a summary of methods and results presented Sect. 7 . 


\section{Cosserat rod model}

In this Section we briefly introduce the Cosserat rod model, which we use for the mechanical description of slender, elastic, 3-dimensional rods [19, 20, 22. The Cosserat rod theory can be seen as a nonlinear, geometrically exact extension of the spatial Timoshenko beam model, and is thus also based on the assumption that the cross-sections remain undeformed, but not necessarily normal to the tangent of the centerline curve, which accounts also for shear deformation.

In the Cosserat model, a rod is represented as a framed curve (see Fig. 2 ) and thus its configuration is completely described by its centerline curve, i.e., the line of its mass centroids $\mathbf{r}:[0, L] \rightarrow \mathbb{R}^{3}$, and a frame, or local orthonormal basis field $\mathbf{R}:[0, L] \rightarrow S O(3)$. The centerline curve is arc-length parameterized in the initial configuration given by $\mathbf{r}_{0}$ and $\mathbf{R}_{0}$, which means that $\left\|\mathbf{r}_{0}^{\prime}(s)\right\|=\left\|\frac{d \mathbf{r}_{0}}{d s}\right\|=1 \forall s \in[0, L]$ and thus $L=\int_{0}^{L}\left\|\mathbf{r}_{0}^{\prime}(s)\right\| d s$ is the length of the curve. The local frames describe the evolution of the orientation of the cross-section and can be associated with 3D rotation matrices $\mathbf{R}(s)=\left(\mathbf{g}_{1}(s), \mathbf{g}_{2}(s), \mathbf{g}_{3}(s)\right) \in \mathbb{R}^{3 \times 3}: \mathbf{R}^{\top} \mathbf{R}=$ $\mathbf{I}$, $\operatorname{det} \mathbf{R}=1 \forall s \in[0, L]$. As in [42], we use unit quaternions, i.e., normalized quadruples of real numbers $\mathbf{q}=\left(q_{1}, q_{2}, q_{3}, q_{4}\right)^{\top} \in \mathbb{R}^{4}:\|\mathbf{q}\|=1$, for the parameterization of frames resp. rotation matrices:

$$
\mathbf{q}:[0, L] \rightarrow S O(3) \leadsto \mathbf{R}(s)=\mathbf{R}(\mathbf{q}(s))
$$

where

$$
\mathbf{R}(\mathbf{q})=\left(\begin{array}{ccc}
q_{1}^{2}-q_{2}^{2}-q_{3}^{2}+q_{4}^{2} & 2\left(q_{1} q_{2}-q_{3} q_{4}\right) & 2\left(q_{1} q_{3}+q_{2} q_{4}\right) \\
2\left(q_{1} q_{2}+q_{3} q_{4}\right) & -q_{1}^{2}+q_{2}^{2}-q_{3}^{2}+q_{4}^{2} & 2\left(q_{2} q_{3}-q_{1} q_{4}\right) \\
2\left(q_{1} q_{3}-q_{2} q_{4}\right) & 2\left(q_{2} q_{3}+q_{1} q_{4}\right) & -q_{1}^{2}-q_{2}^{2}+q_{3}^{2}+q_{4}^{2}
\end{array}\right)
$$

Based on the current (deformed) centerline $\mathbf{r}(s)$ and rotation matrix $\mathbf{R}(s)$ associated with the frame, as well as the initial (undeformed) configuration $\mathbf{r}_{0}(s)$ and $\mathbf{R}_{0}(s)$, the kinematics of the Cosserat rod can be derived. Dropping the dependency on the arc-length parameter $s$ in the notation, the translational strains are given in the initial, material configuration as:

$$
\varepsilon=\mathbf{R}^{\top} \mathbf{r}^{\prime}-\mathbf{R}_{0}^{\top} \mathbf{r}_{0}^{\prime}
$$

Using the curvature vector of the rod:

$$
\mathbf{k}=\left(\begin{array}{l}
\mathbf{g}_{2}^{\prime \top} \mathbf{g}_{3} \\
\mathbf{g}_{3}^{\prime \top} \mathbf{g}_{1} \\
\mathbf{g}_{1}^{\prime \top} \mathbf{g}_{2}
\end{array}\right) \quad \Leftrightarrow \quad[\mathbf{k}]_{\times}=\mathbf{R}^{\prime \top} \mathbf{R}
$$

where $[\cdot]_{\times}$represents the skew-symmetric cross-product matrix, the rotational strains are defined as:

$$
\kappa=\mathbf{k}-\mathbf{k}_{0} .
$$

With these two nonlinear strain vectors and a commonly used linear elastic constitutive law, which restricts the model to large deformations and rotations, but small strains and stresses, the translational and rotational stress resultants in the material configuration can be computed as:

$$
\begin{aligned}
& \sigma=\mathbf{A} \varepsilon+\mathbf{B} \kappa, \\
& \chi=\mathbf{B}^{\top} \varepsilon+\mathbf{C} \kappa .
\end{aligned}
$$

The constitutive matrices $\mathbf{A}, \mathbf{B}, \mathbf{C} \in \mathbb{R}^{3 \times 3}$ depend on the cross-section geometry and material, and will be described in detail in Sect. 4 for spatially varying and functionally graded cross-sections. The stress vectors defined in (6) are now rotated from the local into the global Euclidian coordinate frame, or in other words they are transformed from the material to the spatial configuration:

$$
\begin{aligned}
\mathbf{n} & =\mathbf{R} \sigma, \\
\mathbf{m} & =\mathbf{R} \chi .
\end{aligned}
$$




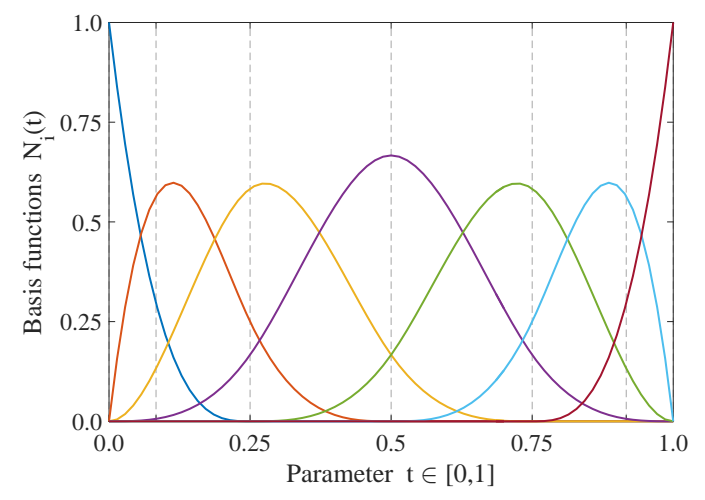

(a) B-Spline basis functions

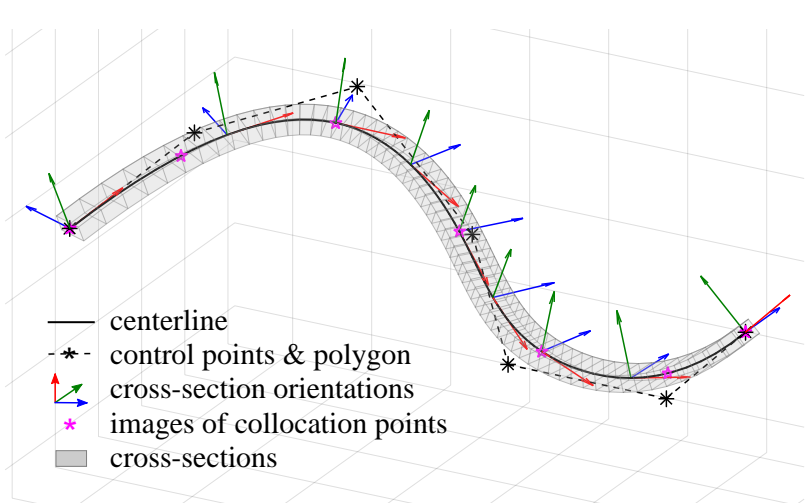

(b) Isogeometric parameterization of rod with B-Spline curve

Figure 3: Isogeometric rod parameterization; cubic B-Spline basis functions as shown in (a) with $p=3, m=11, n=7, \Xi=$ $\left\{0,0,0,0, \frac{1}{4}, \frac{1}{2}, \frac{3}{4}, 1,1,1,1\right\}$ are used for the isogeometric parameterization of the Cosserat rod in (b) with centerline and rotation quaternions as B-Spline curves (see [42])

Finally, the governing equations of the mechanics of the Cosserat rod model are formulated in terms of equilibria of linear and angular momentum in the current, spatial configuration:

$$
\begin{aligned}
\mathbf{n}^{\prime}+\overline{\mathbf{n}}=\mathbf{0} & \forall s \in(0, L), \\
\mathbf{m}^{\prime}+\mathbf{r}^{\prime} \times \mathbf{n}+\overline{\mathbf{m}}=\mathbf{0} & \forall s \in(0, L) .
\end{aligned}
$$

Here $\overline{\mathbf{n}}$ and $\overline{\mathbf{m}}$ are external distributed forces and moments. Furthermore, these differential equations have to be completed with appropriate boundary conditions at the two ends of the rod for $s=0$ and $s=L$. Fixed displacements $\hat{\mathbf{r}}$ and rotations $\hat{\mathbf{q}}$ are specified as Dirichlet boundary conditions $\mathbf{r}(s)=\hat{\mathbf{r}}, \mathbf{q}(s)=\hat{\mathbf{q}}$ at $s=0, L$ and forces $\hat{\mathbf{n}}$ and moments $\hat{\mathbf{m}}$ as Neumann boundary conditions $\mathbf{n}(s)=\hat{\mathbf{n}}, \mathbf{m}(s)=\hat{\mathbf{m}}$ at $s=0, L$. Additionally, a unit length constraint for quaternions must hold to complete the equilibrium equations: $\mathbf{q}^{\top} \mathbf{q}-1=0 \forall s \in[0, L]$.

\section{Isogeometric collocation method}

For the numerical discretization of the Cosserat rod model presented above we employ an isogeometric collocation method. The approach is based on the parameterization of the unknown fields $\mathbf{r}(s)$ and $\mathbf{q}(s)$ using spline functions and the collocation of the equilibrium equations (8). This method was introduced and investigated in [42, and here we briefly review the basic approach.

\subsection{Spline parameterization of Cosserat rods}

The basis of any isogeometric method is the parameterization of geometry and unknowns using B-Splines and Non-Uniform Rational B-Splines (NURBS), which are widely used in computer-aided design [43, 59. Definitions and properties of B-Splines and NURBS can be found in detail in 60. Here, we briefly introduce the main terminology used associated with splines.

B-Splines are piece-wise polynomial functions and Non-Uniform Rational B-Spline (NURBS) piece-wise rational function of degree $p$ and order $p+1$. With $N_{i}(\xi): \Omega_{0} \rightarrow[0,1], i=1, \ldots, n$ we denote the spline (B-Spline or NURBS) basis functions, which are defined on the parameter domain $\Omega_{0}=\left[\xi_{1}, \xi_{m}\right] \subset \mathbb{R}$ using a knot vector $\Xi=\left\{\xi_{1}, \ldots, \xi_{m}\right\}$ with $m=n+p+1$, i.e., a non-decreasing sequence of knots $\xi_{i} \in \mathbb{R}(i=$ $1, \ldots, m), \xi_{i} \leq \xi_{i+1}(i=1, \ldots, m-1)$. For two distinct knots $\xi_{i} \neq \xi_{i+1}$ the half-open interval $\left[\xi_{i}, \xi_{i+1}\right)$ is called the $i$-th knot span or element and the total number of nonzero knot spans or elements in $\Xi$ is denoted by $\ell$. Typically, only open knot vectors are used in IGA, which means that the first and last knot have multiplicity $p+1$, while inner knots have multiplicity $1 \leq k \leq p$. 
The geometry description of a Cosserat rod can now be expressed using spline curves for the initial centerline $\mathbf{r}_{0}$ and rotation quaternions $\mathbf{q}_{0}$ :

$$
\begin{aligned}
& \mathbf{r}_{0}: \Omega_{0} \rightarrow \mathbb{R}^{3}, \quad \mathbf{r}_{0}(\xi)=\sum_{i=1}^{n} N_{i}(\xi) \mathbf{r}_{0, i}, \\
& \mathbf{q}_{0}: \Omega_{0} \rightarrow \mathbb{R}^{4}, \quad \mathbf{q}_{0}(\xi)=\sum_{i=1}^{n} N_{i}(\xi) \mathbf{q}_{0, i}, \quad\left\|\mathbf{q}_{0}(\xi)\right\|=1
\end{aligned}
$$

with control points $\left\{\mathbf{r}_{0, i}\right\}_{i=1, \ldots, n}, \mathbf{r}_{0, i} \in \mathbb{R}^{3}$ and $\left\{\mathbf{q}_{0, i}\right\}_{i=1, \ldots, n}, \mathbf{q}_{0, i} \in \mathbb{R}^{4}$.

For illustration, the parameterization of a rod using cubic B-Spline basis functions $(p=3)$ with $n=7$ control points and $\ell=4$ elements in the knot vector $\Xi=\left\{0,0,0,0, \frac{1}{4}, \frac{1}{2}, \frac{3}{4}, 1,1,1,1\right\}$ is shown in Fig. 3 . Figure $3 \mathrm{a}$ shows the basis functions and Fig. $3 \mathrm{~b}$ the rod itself in terms of its centerline curve and crosssection frames.

Since the centerline is now parameterized as a spline curve $\mathbf{r}_{0}(\xi): \Omega_{0} \rightarrow \mathbb{R}^{3}$ with an arbitrary domain of parameterization $\Omega_{0} \subset \mathbb{R}$, it is general not arc-length parameterized. Thus the derivatives of any vector field $\xi \rightarrow \mathbf{y}(\xi):[0,1] \rightarrow \mathbb{R}^{d}$ required for evaluation of the Cosserat rod model need to be converted to arc-length parameterization using:

$$
\mathbf{y}^{\prime}=\frac{d \mathbf{y}}{d s}=\frac{d \mathbf{y}}{d \xi} \frac{d \xi}{d s}=\dot{\mathbf{y}}\left(\frac{d s}{d \xi}\right)^{-1}=\dot{\mathbf{y}} \frac{1}{\left\|\dot{\mathbf{r}}_{0}(\xi)\right\|}=\frac{1}{J} \dot{\mathbf{y}}
$$

with $\dot{\mathbf{y}}:=d \mathbf{y} / d \xi$ and $J(\xi):=\left\|\dot{\mathbf{r}}_{0}(\xi)\right\|$.

\subsection{Collocation of strong form of equilibrium equations}

As in isoparametric finite elements, the centerline position $\mathbf{r}$ and rotation quaternion $\mathbf{q}$ in the current/deformed configuration are now discretized as spline curves $\mathbf{r}_{h}$ and $\mathbf{q}_{h}$, just like their initial counterparts $\mathbf{r}_{0}$ and $\mathbf{q}_{0}$ in $(9)$ :

$$
\begin{aligned}
& \mathbf{r}_{h}: \Omega_{0} \rightarrow \mathbb{R}^{3}, \quad \mathbf{r}_{h}(\xi)=\sum_{i=1}^{n} N_{i}(\xi) \mathbf{r}_{i}, \\
& \mathbf{q}_{h}: \Omega_{0} \rightarrow \mathbb{R}^{4}, \quad \mathbf{q}_{h}(\xi)=\sum_{i=1}^{n} N_{i}(\xi) \mathbf{q}_{i}, \quad\left\|\mathbf{q}_{h}(\xi)\right\|=1 .
\end{aligned}
$$

The basis functions $N_{i}$ here refer to either the same or $p$ - $/ h$ - $/ k$-refined versions of the ones in $(9)$ and the control points $\mathbf{r}_{i} \in \mathbb{R}^{3}$ and $\mathbf{q}_{i} \in \mathbb{R}^{4}$ are arranged in two vectors $\overrightarrow{\mathbf{r}}=\left(\mathbf{r}_{i}\right)_{i=1, \ldots, n} \in \mathbb{R}^{3 n}$ and $\overrightarrow{\mathbf{q}}=\left(\mathbf{q}_{i}\right)_{i=1, \ldots, n} \in$ $\mathbb{R}^{4 n}$.

Now collocation of the strong form of the equilibrium equations of the Cosserat rod is applied, i.e., the discretization from (11) is substituted into the balance equations $(8)$ and the quaternion normalization condition, which are then evaluated at a set of collocation points $\left\{\tau_{i}\right\}_{i=1, \ldots, n}$ :

$$
\begin{array}{rlrl}
\mathbf{f}_{\mathbf{n}}\left(\tau_{i}\right) & :=\mathbf{n}^{\prime}\left(\tau_{i}\right)+\overline{\mathbf{n}}\left(\tau_{i}\right) & =\mathbf{0}, \\
\mathbf{f}_{\mathbf{m}}\left(\tau_{i}\right) & :=\mathbf{m}^{\prime}\left(\tau_{i}\right)+\mathbf{r}_{h}^{\prime}\left(\tau_{i}\right) \times \mathbf{n}\left(\tau_{i}\right)+\overline{\mathbf{m}}\left(\tau_{i}\right) & =\mathbf{0}, \\
\mathbf{f}_{\mathbf{q}}\left(\tau_{i}\right) & :=\mathbf{q}_{h}\left(\tau_{i}\right)^{\top} \mathbf{q}_{h}\left(\tau_{i}\right)-1 & & =0 .
\end{array}
$$

At the boundary, i.e., for $i=1$ and $i=n$, the above-mentioned equations are replaced with the evaluations of the boundary conditions. In order to guarantee the stability of the method, the collocation points are chosen as the Greville abscissae of the spline knot vector [48, which are defined as:

$$
\tau_{i}=\frac{\xi_{i+1}+\ldots+\xi_{i+p}}{p}, i=1, \ldots, n \text {. }
$$


With 12 we have defined a nonlinear system of $7 n$ equations for the $7 n$ unknowns, i.e., the control point vectors $\overrightarrow{\mathbf{r}}$ for $\mathbf{r}_{h}$ and $\overrightarrow{\mathbf{q}}$ for $\mathbf{q}_{h}$, which we can write as $\mathbf{f}: \mathbb{R}^{3 n} \times \mathbb{R}^{4 n} \rightarrow \mathbb{R}^{7 n}$,

$$
\mathbf{f}(\overrightarrow{\mathbf{r}}, \overrightarrow{\mathbf{q}})=\left(\begin{array}{c}
\mathbf{f}_{\mathrm{n}}\left(\tau_{i}\right) \\
\mathbf{f}_{\mathrm{m}}\left(\tau_{i}\right) \\
\mathbf{f}_{\mathrm{q}}\left(\tau_{i}\right)
\end{array}\right)_{i=1, \ldots, n}(\overrightarrow{\mathbf{r}}, \overrightarrow{\mathbf{q}})=\mathbf{0}
$$

This nonlinear system is then solved with a Newton's method, which requires also the evaluation of the tangent stiffness matrix $\mathbf{K}(\overrightarrow{\mathbf{r}}, \overrightarrow{\mathbf{q}})=d \mathbf{f} / d(\overrightarrow{\mathbf{r}}, \overrightarrow{\mathbf{q}})$ for linearization, see 42 ] for details.

In [42] it is furthermore shown how the isogeometric collocation formulation can be extended to rod structures, where several rods can be interconnected. The rods are coupled rigidly, which means that change of position and rotation have to be equal for all rods connected at an interface and that the equilibria of linear and angular momentum must hold at the interface.

\section{Isogeometric modeling of rods with spatially varying geometric and material parameters}

Typically, the cross-sections of Cosserat rods as introduced above, or in general also in other 2D and 3D beam models, are assumed to be made from a single material and to be constant over the length of the beam. However, the constitutive relationship established in (6) is not restricted to such cases and can also admits transversally (TV) and axially varying (AV) rod cross-sections, where the material composition can change throughout the cross-section and material and geometry can both vary along the centerline. In the following, we address those more general cases through the isogeometric analysis framework using spline parameterizations.

\subsection{Constitutive coefficients for transverse variations}

The constitutive equation (6) defines a linear relationship between strain and stress resultant of the rod model, and reads in its most general formulation as:

$$
\left(\begin{array}{l}
\boldsymbol{\sigma} \\
\chi
\end{array}\right)=\left(\begin{array}{cc}
\mathbf{A} & \mathbf{B} \\
\mathbf{B}^{\top} & \mathbf{C}
\end{array}\right)\left(\begin{array}{l}
\varepsilon \\
\kappa
\end{array}\right)
$$

where the constitutive matrices $\mathbf{A}, \mathbf{B}, \mathbf{C} \in \mathbb{R}^{3 \times 3}$ take the following form:

$$
\mathbf{A}=\left(\begin{array}{ccc}
A_{11} & A_{12} & 0 \\
A_{12} & A_{22} & 0 \\
0 & 0 & A_{33}
\end{array}\right), \quad \mathbf{B}=\left(\begin{array}{ccc}
0 & 0 & B_{13} \\
0 & 0 & B_{23} \\
B_{31} & B_{32} & 0
\end{array}\right), \quad \mathbf{C}=\left(\begin{array}{ccc}
C_{11} & C_{12} & 0 \\
C_{12} & C_{22} & 0 \\
0 & 0 & C_{33}
\end{array}\right)
$$

Their coefficients depend on the geometric layout and material constitution of the beam cross-section. A detailed derivation of the coefficients can be found in [25] and takes into account that the Bernoulli hypothesis must be fulfilled, i.e., that plain cross-sections must remain plain under deformation.

In the following, we restrict ourselves to cases where $A_{12}=B_{13}=B_{23}=0$ and according to [25] the remaining coefficients can be computed as:

$$
\begin{aligned}
& A_{11}=k_{1} \int_{S} G(\mathbf{x}) d \mathbf{x}, \quad A_{22}=k_{2} \int_{S} G(\mathbf{x}) d \mathbf{x}, \quad A_{33}=\int_{S} E(\mathbf{x}) d \mathbf{x} \\
& B_{31}=\int_{S} E(\mathbf{x}) x_{2} d \mathbf{x}, \quad B_{32}=\int_{S} E(\mathbf{x}) x_{1} d \mathbf{x}, \quad C_{11}=\int_{S} E(\mathbf{x}) x_{2}^{2} d \mathbf{x}, \\
& C_{22}=\int_{S} E(\mathbf{x}) x_{1}^{2} d \mathbf{x}, \quad C_{12}=\int_{S} E(\mathbf{x}) x_{1} x_{2} d \mathbf{x}, \quad C_{33} \equiv C_{33}(S, E) .
\end{aligned}
$$

Here, $k_{1}$ and $k_{2}$ are the shear correction factors, $S$ is the geometric domain of the cross-section, and $G=$ $E /(2+2 \nu)$ and $E$ are the shear and Young's modulus and $\nu$ is the Poisson's ratio, which all depend on the 


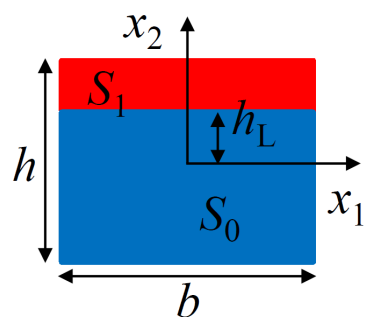

(a) rectangular bilayer

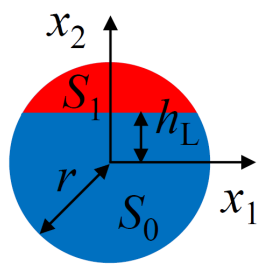

(b) circular bilayer

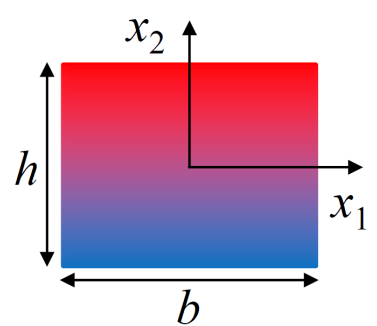

(c) continuously graded

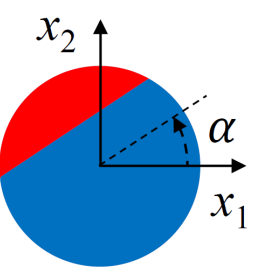

(d) rotated

Figure 4: Definition and geometric parameters of transversally varying (functionally graded) cross-section types: (a) rectangular and (b) circular bilayer cross-sections, (c) rectangular continuously graded cross-section (here linearly graded), and (d) rotated cross-section (here circular bilayer)

material point $\mathbf{x} \in S$ inside the cross-section domain $S$, which is aligned such that $x_{1}$ denotes the coordinate along the $\mathbf{g}_{1}$-direction and $x_{2}$ along the $\mathbf{g}_{2}$-direction.

For the conventional case of homogeneous, isotropic cross-sections, where $E$ and $G$ are constant throughout the cross-section, this simplifies to:

$$
\mathbf{A}=\left(\begin{array}{ccc}
k_{1} G A & 0 & 0 \\
0 & k_{2} G A & 0 \\
0 & 0 & E A
\end{array}\right), \quad \mathbf{B} \equiv \mathbf{0}, \quad \mathbf{C}=\left(\begin{array}{ccc}
E I_{1} & 0 & 0 \\
0 & E I_{2} & 0 \\
0 & 0 & G J
\end{array}\right),
$$

where $A$ is the area, $I_{1}$ and $I_{2}$ the second moments of area, and $J$ the polar moment of area of $S$.

\subsubsection{Bilayer laminate cross-sections}

As a first type of laminate or composite cross-section, we present the coefficients for bilayer cross-sections with either rectangular or circular shape, see Fig. $4 \mathrm{a}$ b

For the following derivations, a few basic definitions and assumptions are made: the geometric dimensions of the cross-section $S \subset \mathbb{R}^{2}$ are width $b$ and height $h$ for rectangular and radius $r$ for circular shape; the layers are separated along the local $\mathbf{g}_{2}$-direction; the layering is determined by the layer height $h_{L} \in(-h / 2, h / 2)$ or $h_{L} \in(-r, r)$, respectively; the domain of the lower layer is denoted as $S_{0}=\left\{\mathbf{x} \in S: x_{2} \leq h_{L}\right\}$ with Young's modulus $E_{0}$; the domain of the upper layer is denoted as $S_{1}=\left\{\mathbf{x} \in S: x_{2}>h_{L}\right\}$ with Young's modulus $E_{1}$; for both layers Poisson's ratios $\nu=\nu_{0}=\nu_{1}$ and mass densities $\rho=\rho_{0}=\rho_{1}$ are equal.

Then, the constitutive matrices can be written as:

$$
\mathbf{A}=\left(\begin{array}{ccc}
k_{1} \bar{\mu} & 0 & 0 \\
0 & k_{2} \bar{\mu} & 0 \\
0 & 0 & 1
\end{array}\right) A_{33}, \quad \mathbf{B}=\left(\begin{array}{ccc}
0 & 0 & 0 \\
0 & 0 & 0 \\
B_{31} & 0 & 0
\end{array}\right), \quad \mathbf{C}=\left(\begin{array}{ccc}
C_{11} & 0 & 0 \\
0 & C_{22} & 0 \\
0 & 0 & C_{33}
\end{array}\right)
$$

with $k_{1}=k_{2}=\frac{5}{6}, \bar{\mu}=\frac{1}{2(1+\nu)}$, and:

$$
\begin{array}{lll}
A_{33}=E_{0} \bar{A}_{33}^{0}+E_{1} \bar{A}_{33}^{1}, & \bar{A}_{33}^{0}=\int_{S_{0}} d \mathbf{x}, & \bar{A}_{33}^{1}=\int_{S_{1}} d \mathbf{x}, \\
B_{31}=E_{0} \bar{B}_{31}^{0}+E_{1} \bar{B}_{31}^{1}, & \bar{B}_{31}^{0}=\int_{S_{0}} x_{2} d \mathbf{x}, & \bar{B}_{31}^{1}=\int_{S_{1}} x_{2} d \mathbf{x}, \\
C_{11}=E_{0} \bar{C}_{11}^{0}+E_{1} \bar{C}_{11}^{1}, & \bar{C}_{11}^{0}=\int_{S_{0}} x_{2}^{2} d \mathbf{x}, & \bar{C}_{11}^{1}=\int_{S_{1}} x_{2}^{2} d \mathbf{x}, \\
C_{22}=E_{0} \bar{C}_{22}^{0}+E_{1} \bar{C}_{22}^{1}, & \bar{C}_{22}^{0}=\int_{S_{0}} x_{1}^{2} d \mathbf{x}, & \bar{C}_{22}^{1}=\int_{S_{1}} x_{1}^{2} d \mathbf{x}, \\
C_{33} \equiv C_{33}\left(S_{0}, E_{0}, S_{1}, E_{1}\right) . &
\end{array}
$$


The detailed calculation of those coefficients and integrals for both cases of rectangular and circular bilayer cross-sections, please refer to the Appendix A.1.

\subsubsection{Continuously varying cross-sections}

Next, we introduce the commonly addressed case of a rectangular, continuously functionally graded cross-section, see Fig. 4c

Here, the Young's modulus varies throughout the cross-section as a function of the $x_{2}$-coordinate:

$$
E\left(x_{2}\right)=E_{0}+\left(E_{1}-E_{0}\right)\left(\frac{1}{2}+\frac{x_{2}}{h}\right)^{p}
$$

where $x_{2} \in[-h / 2, h / 2]$ and $p>0$ is the exponent of a power law. This yields an asymmetric Young's modulus distribution with respect to the $x_{1}$-axis of the cross-section and thus to the same general representation of the constitutive matrices as in $(19)$ and 20 . The detailed coefficients are derived and calculated in Appendix A.2.

\subsubsection{Rotated cross-sections}

To further generalize the transverse variation of cross-section types we now introduce rotated crosssections, where for instance the orientation of the layering or gradient described in the preceding subsections is rotated by an angle $\alpha \in \mathbb{R}$ around the local $\mathbf{g}_{3}$-axis, see Fig. $4 \mathrm{~d}$.

For any point $\mathbf{x}=\left(x_{1}, x_{2}\right)^{\top} \in S$, where $S$ is the rotated version of an axis-aligned cross-section $S^{0}$ with layered or graded Young's modulus distribution $E\left(\mathbf{x}^{0}\right)$, this means that:

$$
\mathbf{x}=\varphi\left(\mathbf{x}^{0}\right)=\mathbf{R}_{\alpha} \mathbf{x}^{0}=\left(\begin{array}{cc}
\cos \alpha & -\sin \alpha \\
\sin \alpha & \cos \alpha
\end{array}\right) \mathbf{x}^{0}
$$

where $\varphi: S^{0} \rightarrow S$ is an injective and differentiable mapping. Then we can compute the constitutive coefficients of the rotated cross-section $S=\varphi\left(S^{0}\right)$ using integration by substitution with $|\operatorname{det} D \varphi|=\left|\operatorname{det} \mathbf{R}_{\alpha}\right|=1$ :

$$
\begin{aligned}
A_{33}= & \int_{S} E(\mathbf{x}) d \mathbf{x}=\int_{S^{0}} E\left(\mathbf{x}^{0}\right) d \mathbf{x}^{0}=A_{33}^{0}, \\
B_{31}= & \int_{S} E(\mathbf{x}) x_{2} d \mathbf{x}=\int_{S^{0}} E\left(\mathbf{x}^{0}\right)\left(x_{1}^{0} \sin \alpha+x_{2}^{0} \cos \alpha\right) d \mathbf{x}^{0}=\cos \alpha B_{31}^{0}-\sin \alpha B_{32}^{0}, \\
B_{32}= & -\int_{S} E(\mathbf{x}) x_{1} d \mathbf{x}=-\int_{S^{0}} E\left(\mathbf{x}^{0}\right)\left(x_{1}^{0} \cos \alpha-x_{2}^{0} \sin \alpha\right) d \mathbf{x}^{0}=\sin \alpha B_{31}^{0}+\cos \alpha B_{32}^{0}, \\
C_{11}= & \int_{S} E(\mathbf{x}) x_{2}^{2} d \mathbf{x}=\int_{S^{0}} E\left(\mathbf{x}^{0}\right)\left(x_{1}^{0} \sin \alpha+x_{2}^{0} \cos \alpha\right)^{2} d \mathbf{x}^{0}=\cos ^{2} \alpha C_{11}^{0}-2 \cos \alpha \sin \alpha C_{12}^{0}+\sin ^{2} \alpha C_{22}^{0}, \\
C_{22}= & \int_{S} E(\mathbf{x}) x_{1}^{2} d \mathbf{x}=\int_{S^{0}} E\left(\mathbf{x}^{0}\right)\left(x_{1}^{0} \cos \alpha-x_{2}^{0} \sin \alpha\right)^{2} d \mathbf{x}^{0}=\sin ^{2} \alpha C_{11}^{0}+2 \cos \alpha \sin \alpha C_{12}^{0}+\cos ^{2} \alpha C_{22}^{0}, \\
C_{12}= & -\int_{S} E(\mathbf{x}) x_{1} x_{2} d \mathbf{x}=-\int_{S^{0}} E\left(\mathbf{x}^{0}\right)\left(x_{1}^{0} \cos \alpha-x_{2}^{0} \sin \alpha\right)\left(x_{1}^{0} \sin \alpha+x_{2}^{0} \cos \alpha\right) d \mathbf{x}^{0} \\
= & \cos \alpha \sin \alpha\left(C_{11}^{0}-C_{22}^{0}\right)+\left(\cos ^{2} \alpha-\sin ^{2} \alpha\right) C_{12}^{0}, \\
C_{33}= & C_{33}^{0} .
\end{aligned}
$$

More compactly, we can write in matrix form:

$$
\mathbf{A}=\mathbf{A}^{0}\left(=\mathbf{R}_{\alpha} \mathbf{A}^{0} \mathbf{R}_{\alpha}^{\top}\right), \quad \mathbf{B}=\mathbf{B}^{0} \mathbf{R}_{\alpha}^{\top}\left(=\mathbf{R}_{\alpha} \mathbf{B}^{0} \mathbf{R}_{\alpha}^{\top}\right), \quad \mathbf{C}=\mathbf{R}_{\alpha} \mathbf{C}^{0} \mathbf{R}_{\alpha}^{\top},
$$


slightly abusing the notation since $\mathbf{R}_{\alpha}$ is now the extended 3-dimensional rotation matrix:

$$
\mathbf{R}_{\alpha}=\left(\begin{array}{ccc}
\cos \alpha & -\sin \alpha & 0 \\
\sin \alpha & \cos \alpha & 0 \\
0 & 0 & 1
\end{array}\right)
$$

\subsection{Isogeometric parameterization of axially varying cross-section parameters}

As defined earlier, an axially varying beam is characterized by varying properties of the cross-section along the centerline of the beam, see Fig. 2b. In general, we can consider both material and geometric parameters as axially varying. This means that properties such as Young's modulus $E$, Poisson's ratio $\nu$, radius $r$ of a circular cross-section, layer height $h_{L}$ of a bilayer cross-section, or even the general cross-section shape $S$ can be expressed as a function of the arc-length parameter $s$ of the rod.

Summarizing all those cross-section parameters in one vector $\mathbf{u}=(E, \nu, r, \ldots)^{\top} \in \mathbb{R}^{d_{u}}$, we can parameterize the axial variation of the cross-section properties as a NURBS curve:

$$
\mathbf{u}(s)=\sum_{i=1}^{n_{u}} N_{i}^{u}(s) \mathbf{u}_{i} .
$$

In this isogeometric parameterization, $d_{u}$ is the number of design parameters, $N_{i}^{u}$ are $n_{u}$ NURBS basis functions with knot vector $\Xi^{u}$ of degree $p^{u}$ with $\ell^{u}$ elements, and $\mathbf{u}_{i}$ are the $n_{u}$ control points of the curve. This NURBS basis can be chosen arbitrarily, depending on the design of the axially varying rod, i.e., it does not necessarily have to be the same as the one used to parameterize and discretize the rods in (9) or (11).

For the constitutive matrices and coefficients, as introduced above in Sect. 4.1 for both homogeneous and transversally varying cross-sections, the parameterization of the cross-section properties implies, of course, that they also become dependent on $s$ :

$$
\mathbf{A}(s) \equiv \mathbf{A}(\mathbf{u}(s)), \quad \mathbf{B}(s) \equiv \mathbf{B}(\mathbf{u}(s)), \quad \mathbf{C}(s) \equiv \mathbf{C}(\mathbf{u}(s)) .
$$

The axially varying constitutive matrices are used in the constitutive law (6) to compute the stress resultants from the strains, which are then transformed to the material configuration in (7) and finally used in the equilibrium equations (8). Since the balance equations require the arc-length derivation of the internal forces and moments:

$$
\begin{aligned}
\mathbf{n}^{\prime} & =(\mathbf{R} \boldsymbol{\sigma})^{\prime}=\mathbf{R}^{\prime} \boldsymbol{\sigma}+\mathbf{R} \boldsymbol{\sigma}^{\prime}, \\
\mathbf{m}^{\prime} & =(\mathbf{R} \boldsymbol{\chi})^{\prime}=\mathbf{R}^{\prime} \boldsymbol{\chi}+\mathbf{R} \boldsymbol{\chi}^{\prime},
\end{aligned}
$$

it follows for an axially varying rod that also the constitutive matrices have to be differentiated, since they are not constant anymore:

$$
\begin{array}{ll}
\boldsymbol{\sigma}^{\prime}=(\mathbf{A} \boldsymbol{\varepsilon}+\mathbf{B} \boldsymbol{\kappa})^{\prime} & =\mathbf{A}^{\prime} \boldsymbol{\varepsilon}+\mathbf{A} \boldsymbol{\varepsilon}^{\prime}+\mathbf{B}^{\prime} \boldsymbol{\kappa}+\mathbf{B} \boldsymbol{\kappa}^{\prime}, \\
\chi^{\prime}=\left(\mathbf{B}^{\top} \boldsymbol{\varepsilon}+\mathbf{C} \boldsymbol{\kappa}\right)^{\prime} & =\mathbf{B}^{\prime \top} \boldsymbol{\varepsilon}+\mathbf{B}^{\top} \boldsymbol{\varepsilon}^{\prime}+\mathbf{C}^{\prime} \boldsymbol{\kappa}+\mathbf{C}^{\prime} .
\end{array}
$$

Since the constitutive matrices are computed from the vector $\mathbf{u}(s)$, their derivatives $\mathbf{A}^{\prime}, \mathbf{B}^{\prime}$ and $\mathbf{C}^{\prime}$ can be easily evaluated through the isogeometric parameterization, e.g.:

$$
\mathbf{A}^{\prime}=\frac{d \mathbf{A}}{d s}=\sum_{i=1}^{d_{u}} \frac{d \mathbf{A}}{d u_{i}} u_{i}^{\prime} .
$$

\section{Mixed method for slender composite rods}

Shear locking is a well-known phenomenon that occurs when shear compliant beam theories are employed for slender beams, i.e., beams with small thickness-to-length ratios $(r / L \ll 1)$. It manifests itself through 
instability of the numerical method, loss of accuracy and deterioration of convergence rates. Here, we address this issue in the context of rods with transversally varying, functionally graded cross-sections.

A mixed method that cures shear locking for isogeometric collocation of the Timoshenko beam model was already introduced in [51] and theoretically investigated in 53. The approach was also extended to the Cosserat rod model in [42, 61] and is based on an independent discretization of the internal forces $\mathbf{n}$ and moments m:

$$
\mathbf{n}_{h}: \Omega_{0} \rightarrow \mathbb{R}^{3}: \mathbf{n}_{h}(\xi)=\sum_{i=1}^{n} N_{i}(\xi) \mathbf{n}_{i}, \quad \mathbf{m}_{h}: \Omega_{0} \rightarrow \mathbb{R}^{3}: \mathbf{m}_{h}(\xi)=\sum_{i=1}^{n} N_{i}(\xi) \mathbf{m}_{i},
$$

thus introducing additional unknowns with the force and moment control points $\mathbf{n}_{i}$ and $\mathbf{m}_{i}(i=1, \ldots, n)$, and consequently also requiring modified and additional collocated balance equations:

$$
\begin{array}{rlrl}
\mathbf{f}_{\mathbf{n}}\left(\tau_{i}\right): & :=\mathbf{n}_{h}^{\prime}\left(\tau_{i}\right)+\overline{\mathbf{n}}\left(\tau_{i}\right) & =\mathbf{0}, \\
\mathbf{f}_{\mathrm{m}}\left(\tau_{i}\right):=\mathbf{m}_{h}^{\prime}\left(\tau_{i}\right)+\mathbf{r}_{h}^{\prime}\left(\tau_{i}\right) \times \mathbf{n}_{h}\left(\tau_{i}\right)+\overline{\mathbf{m}}\left(\tau_{i}\right) & =\mathbf{0}, \\
\mathbf{f}_{\mathbf{q}}\left(\tau_{i}\right):=\mathbf{q}_{h}\left(\tau_{i}\right)^{\top} \mathbf{q}_{h}\left(\tau_{i}\right)-1 & & =0, \\
\mathbf{f}_{\mathrm{u}}\left(\tau_{i}\right) & :=\mathbf{n}_{h}\left(\tau_{i}\right)-(\mathbf{R} \boldsymbol{\sigma})\left(\tau_{i}\right) & & =\mathbf{0}, \\
\mathbf{f}_{\mathrm{v}}\left(\tau_{i}\right) & :=\mathbf{m}_{h}\left(\tau_{i}\right)-(\mathbf{R} \boldsymbol{\chi})\left(\tau_{i}\right) & & =\mathbf{0} .
\end{array}
$$

Compared to the primal formulation using only displacement and rotation degrees of freedom in $(12)$, here the independent forces $\mathbf{n}_{h}$ and moments $\mathbf{m}_{h}$ are used in the equations $\mathbf{f}_{\mathbf{n}}$ and $\mathbf{f}_{\mathbf{m}}$ and in the additional equations $\mathbf{f}_{\mathrm{u}}$ and $\mathbf{f}_{\mathrm{v}}$ they are set equal to their counterparts $\mathbf{n}=\mathbf{R} \boldsymbol{\sigma}$ and $\mathbf{m}=\mathbf{R} \boldsymbol{\chi}$ computed from the primary variables.

This approach cures the shear locking problem for beams with homogeneous cross-sections, since the ill-conditioning of stress components $\boldsymbol{\sigma}=\mathbf{A} \varepsilon$ and $\chi=\mathbf{C} \boldsymbol{\kappa}$, which is caused by the difference in scales between the constitutive matrices $\mathbf{A} \sim r^{2}$ and $\mathbf{C} \sim r^{4}$, is resolved by the independent discretization of forces $\mathbf{n}_{h}=\mathbf{R} \boldsymbol{\sigma}$ and moments $\mathbf{m}_{h}=\mathbf{R} \boldsymbol{\chi}$. However, when transversally varying, non-symmetric crosssections are used, the constitutive equations additionally include the translational-rotational coupling terms with the matrix $\mathbf{B}$, which scales as $\mathbf{B} \sim r^{3}$, see A.2. This introduces an additional imbalance of the scales within the constitutive equations for both, the translational and rotational stresses. Thus, shear locking and numerical instability can still be present when the above-mentioned mixed method is used for slender, functionally graded rods, see numerical examples in Sect.6.1.

To cure shear locking also for these cases, we introduce an enhanced mixed method, in which the translational and rotational strain contributions to the internal stresses are separated:

$$
\boldsymbol{\sigma}^{\varepsilon}:=\mathbf{A} \varepsilon, \quad \boldsymbol{\sigma}^{\kappa}:=\mathbf{B} \kappa, \quad \chi^{\varepsilon}:=\mathbf{B}^{\top} \varepsilon, \quad \chi^{\kappa}:=\mathbf{C} \kappa .
$$

Then, the corresponding internal force and moment contributions are discretized independently:

$$
\begin{array}{rrrl}
\mathbf{n}_{h}^{\varepsilon}: \Omega_{0} \rightarrow \mathbb{R}^{3}: & \mathbf{n}_{h}^{\varepsilon}(\xi)=\sum_{i=1}^{n} N_{i}(\xi) \mathbf{n}_{i}^{\varepsilon}, & \mathbf{n}_{h}^{\kappa}: \Omega_{0} \rightarrow \mathbb{R}^{3}: & \mathbf{n}_{h}^{\kappa}(\xi)=\sum_{i=1}^{n} N_{i}(\xi) \mathbf{n}_{i}^{\kappa}, \\
\mathbf{m}_{h}^{\varepsilon}: \Omega_{0} \rightarrow \mathbb{R}^{3}: & \mathbf{m}_{h}^{\varepsilon}(\xi)=\sum_{i=1}^{n} N_{i}(\xi) \mathbf{m}_{i}^{\varepsilon}, & \mathbf{m}_{h}^{\kappa}: \Omega_{0} \rightarrow \mathbb{R}^{3}: & \mathbf{m}_{h}^{\kappa}(\xi)=\sum_{i=1}^{n} N_{i}(\xi) \mathbf{m}_{i}^{\kappa} .
\end{array}
$$

In addition to the $7 n$ primal unknowns, i.e., the control points for $\mathbf{r}_{h}$ and $\mathbf{q}_{h}$, we know have $4 \cdot 3 n$ unknowns for the control points $\left\{\mathbf{n}_{i}^{\varepsilon}, \mathbf{n}_{i}^{\kappa}, \mathbf{m}_{i}^{\varepsilon}, \mathbf{m}_{i}^{\kappa}\right\}_{i=1, \ldots, n}$ and the corresponding collocated balance equations become: 


$$
\begin{array}{rlrl}
\mathbf{f}_{\mathbf{n}}\left(\tau_{i}\right):=\left(\mathbf{n}_{h}^{\varepsilon}\left(\tau_{i}\right)+\mathbf{n}_{h}^{\kappa}\left(\tau_{i}\right)\right)^{\prime}+\overline{\mathbf{n}}\left(\tau_{i}\right) & =\mathbf{0}, \\
\mathbf{f}_{\mathbf{m}}\left(\tau_{i}\right):=\left(\mathbf{m}_{h}^{\varepsilon}\left(\tau_{i}\right)+\mathbf{m}_{h}^{\kappa}\left(\tau_{i}\right)\right)^{\prime}+\mathbf{r}_{h}^{\prime}\left(\tau_{i}\right) \times\left(\mathbf{n}_{h}^{\varepsilon}\left(\tau_{i}\right)+\mathbf{n}_{h}^{\kappa}\left(\tau_{i}\right)\right)+\overline{\mathbf{m}}\left(\tau_{i}\right) & =\mathbf{0}, \\
\mathbf{f}_{\mathbf{q}}\left(\tau_{i}\right):=\mathbf{q}_{h}\left(\tau_{i}\right)^{\top} \mathbf{q}_{h}\left(\tau_{i}\right)-1 & & =0, \\
\mathbf{f}_{\mathbf{u}}^{\varepsilon}\left(\tau_{i}\right): & =\mathbf{n}_{h}^{\varepsilon}\left(\tau_{i}\right)-\left(\mathbf{R} \boldsymbol{\sigma}^{\varepsilon}\right)\left(\tau_{i}\right) & & =\mathbf{0}, \\
\mathbf{f}_{\mathrm{u}}^{\kappa}\left(\tau_{i}\right) & :=\mathbf{n}_{h}^{\kappa}\left(\tau_{i}\right)-\left(\mathbf{R} \boldsymbol{\chi}^{\kappa}\right)\left(\tau_{i}\right) & & =\mathbf{0}, \\
\mathbf{f}_{\mathrm{v}}^{\varepsilon}\left(\tau_{i}\right) & :=\mathbf{m}_{h}^{\varepsilon}\left(\tau_{i}\right)-\left(\mathbf{R} \boldsymbol{\sigma}^{\varepsilon}\right)\left(\tau_{i}\right) & & =\mathbf{0}, \\
\mathbf{f}_{\mathrm{v}}^{\kappa}\left(\tau_{i}\right) & :=\mathbf{m}_{h}^{\kappa}\left(\tau_{i}\right)-\left(\mathbf{R} \boldsymbol{\chi}^{\kappa}\right)\left(\tau_{i}\right) & & =\mathbf{0} .
\end{array}
$$

Altogether, 35 forms a nonlinear system of $19 n$ equations and unknowns:

$$
\mathbf{f}\left(\overrightarrow{\mathbf{r}}, \overrightarrow{\mathbf{q}}, \overrightarrow{\mathbf{n}}^{\varepsilon}, \overrightarrow{\mathbf{n}}^{\kappa}, \overrightarrow{\mathbf{m}}^{\varepsilon}, \overrightarrow{\mathbf{m}}^{\kappa}\right)=\left(\left(\mathbf{f}_{\mathrm{n}}, \mathbf{f}_{\mathrm{m}}, \mathbf{f}_{\mathbf{q}}, \mathbf{f}_{\mathrm{u}}^{\varepsilon}, \mathbf{f}_{\mathrm{u}}^{\kappa}, \mathbf{f}_{\mathrm{v}}^{\varepsilon}, \mathbf{f}_{\mathrm{v}}^{\kappa}\right)\left(\tau_{i}\right)\right)_{i=1, \ldots, n}^{\top}=\mathbf{0} .
$$

This nonlinear system is again solved with a Newton's method.

To achieve optimal convergence rates, the same basis functions $N_{i}$ are used for the discretization of $\mathbf{n}_{h}^{\varepsilon}, \mathbf{n}_{h}^{\kappa}, \mathbf{m}_{h}^{\varepsilon}$ and $\mathbf{m}_{h}^{\kappa}$ as were used for $\mathbf{r}_{h}$ and $\mathbf{q}_{h}$ and all equations are collocated at the original collocation points $\tau_{i}$ [51]. Furthermore, much like the initial mixed method shown in (31), the continuity requirements for the basis functions are reduced, since both, the primal and secondary unknowns, only have to be differentiated once instead of twice, as in the original method (12). This also reduces the computational effort for the assembly of the system and its Jacobian matrix, since second derivatives do not have to be evaluated anymore. The split of translational and rotational parts of internal forces and moments in the enhanced mixed method introduces additional computational effort, since more discretized variables need to be evaluated (e.g., $\mathbf{n}_{h}^{\varepsilon}$ and $\mathbf{n}_{h}^{\kappa}$ instead of $\mathbf{n}_{h}$ ), transformed (e.g., $\mathbf{R} \boldsymbol{\sigma}^{\varepsilon}$ and $\mathbf{R} \boldsymbol{\sigma}^{\kappa}$ instead of $\mathbf{R} \boldsymbol{\sigma}$ ), and assembled into separate positions in the force vector and stiffness matrix. Also, the computational effort for solving the linear systems within the Newton's method increases, of course, since the system size increases from $7 n$ for the primal method and $13 n$ for the simpler mixed method to $19 n$ for the enhanced mixed method.

However, the main objective and advantage of the mixed methods, and particularly the novel enhanced mixed method for transversally varying cross-sections introduced here, is that they cure shear locking and thus provide higher accuracy and increased stability compared to the primal or simple mixed method. This means that a smaller degree or number of elements can be used to obtain same accuracy and less load steps and less iterations per load step are required to obtain convergence, which makes the mixed methods overall computationally much more efficient, as will be shown in Sect. 6.1.2

\section{Numerical results}

In this Section we apply isogeometric parameterizations of axially and transversally varying cross-sections together with the isogeometric collocation methods for nonlinear 3D composite rods in several computational examples. We focus on both, the validation of the approach using convergence studies and reference examples, as well as advanced applications that highlight the capabilities and efficiency of the isogeometric modeling and analysis concept.

\subsection{Convergence studies}

First, we investigate the effect of employing axially varying parameters and transversally varying, functionally graded cross-sections on the general accuracy and convergence behavior of the isogeometric collocation methods presented.

\subsubsection{Axially varying cross-section parameters}

As reference set-up, we take a rod of length $L=1.0 \mathrm{~m}$ with a circular cross-section of radius $R=0.05$ $\mathrm{m}$. The initial, constant material parameters are Young's modulus $E(s) \equiv E_{0}=100 \mathrm{MPa}$ and Poisson's 


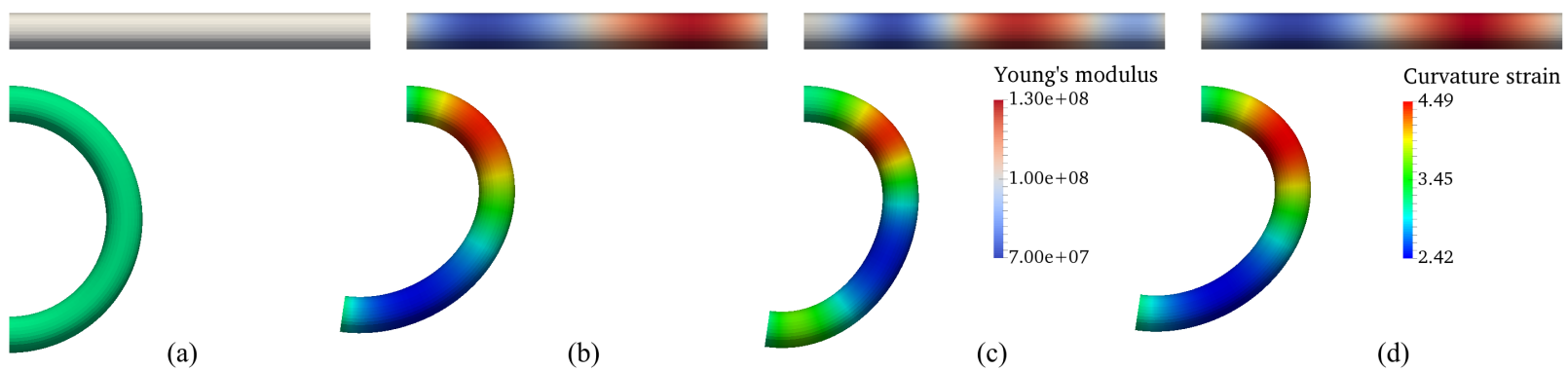

Figure 5: Rods with axially varying material parameters. Initial rods with axially varying Young's modulus distributions on top (colored by Young's modulus) and deformed configurations below (colored by curvature strain)

ratio $\nu=0.45$, which we refer to as case (a). The rod is clamped at one end and a moment $\hat{m}(L)=\pi E_{0} I$ is applied at the other over 8 load steps, deforming it into a semicircle, see Fig. 5 a. Now, we introduce three variations of the above-mentioned rod with axially varying Young's modulus, which are parameterized as follows using $\mathbf{u}(s) \equiv E(s)$, see also Fig. 5 p-d:

(b) $p=3, \ell=1, n=4, \overrightarrow{\mathbf{u}}=E_{0} \cdot(1.0,0.0,2.0,1.0)$. The Young's modulus is parameterized globally along the rod as a polynomial of degree 3 , since the B-Spline has only 1 element.

(c) $p=3, \ell=4, n=7, \overrightarrow{\mathbf{u}}=E_{0} \cdot(1.0,1.0,0.4,1.4,1.2,0.7,1.0)$. The Young's modulus is parameterized arbitrarily along the rod as a B-Spline of degree 3 with 4 elements (knot spans).

(d) The Young's modulus is parameterized locally, using the same B-Spline basis employed for the spatial discretization of the rod and $\overrightarrow{\mathbf{u}}$ is chosen such that $E(s)$ interpolates a sinusoidal curve, i.e., $E\left(\tau_{i}\right)=$ $E_{0} \cdot\left(1-0.3 \sin 2 \pi \tau_{i}\right)$.

For all four cases of Young's modulus parameterizations, we apply the same moment to deform them and compute a reference solution $\mathbf{r}_{*}$ with a highly refined isogeometric discretization using $p=10, \ell=128$. Then, we evaluate the $L^{2}$-errors of deformed centerline positions $e_{h}=\left\|\mathbf{r}_{h}-\mathbf{r}_{*}\right\|$, where $\mathbf{r}_{h}$ indicates the solutions with lower levels of $k$-refinement obtained for $p=3, \ldots, 8$ and $\ell=4, \ldots, 64$. The corresponding convergence plots for $e_{h}$ over number of elements (knot spans) $\ell$ are shown in Fig. 6.

Figure 6a refers to the conventional case of a rod with constant cross-section parameters, i.e., here constant Young's modulus. The convergence rates that can be observed here correspond to the typical ones to be expected for an isogeometric collocation method, as already presented for Cosserat rods in [42.

For the global parameterization with degree 3, which is shown in Fig. 6b, the convergence rates are well reproduced, but the error constants are about 1-3 orders of magnitude higher due to the variation of the Young's modulus. This shows that the collocation method can deal well with those global cross-section parameterizations and there is no negative effect on convergence rates. Such behavior can be expected for a Galerkin method, since the element-wise integration can also exactly integrate the axially varying material parameters (if the degree of the spatial discretization is not less than the one of the material parameterization). However, in the case of a collocation method, where there is no fixed distribution of collocation points over elements, such behavior is not immediately clear from the theoretical point of view.

When the arbitrary parameterization over 4 elements with degree 3 is applied, see Fig. 6c, a negative effect on both error constants as well as convergence rates of higher order discretizations with $p \geq 6$ can be observed, since the collocation points of the spatial discretization are not compatible with the Young's modulus parameterization. Furthermore, the absolute errors are again higher, since the axially varying parameterization of the cross-section is "rougher" due to the choice of control points in $\overrightarrow{\mathbf{u}}$.

The Young's modulus distribution in case (d) is actually very similar to case (b), but interpolating the sinusoidal shape of $E(s)$ with the same basis functions as used in the spatial discretizations. As shown in the convergence plots in Fig. 6d, the absolute errors are also similar to (b), however, especially for higher order and higher number of elements it seems that the theoretical convergence rates can be more closely 


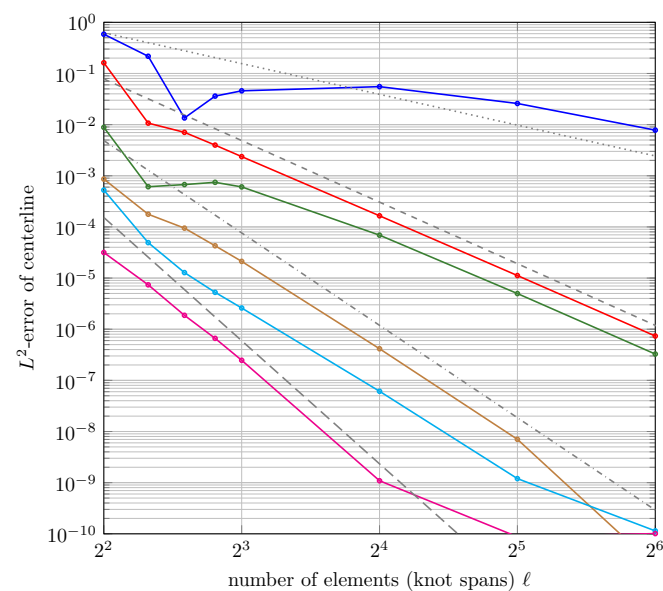

(a) $E(s)=E_{0}$ is constant

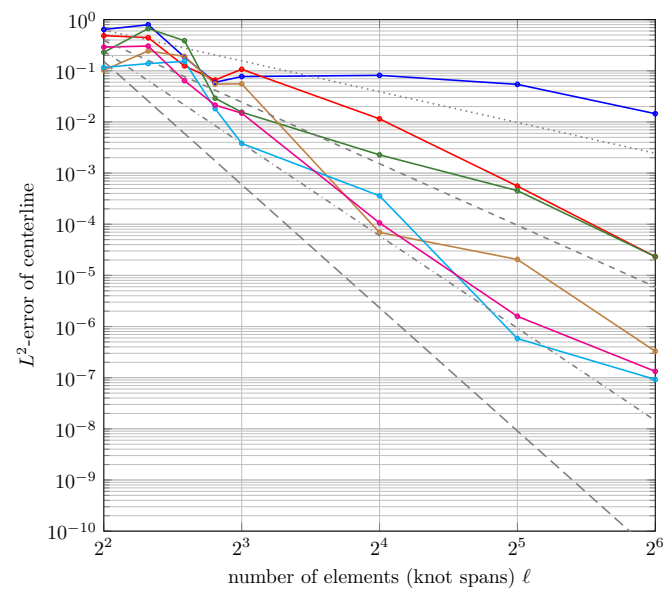

(c) $E(s)$ of degree 3 with 4 elements
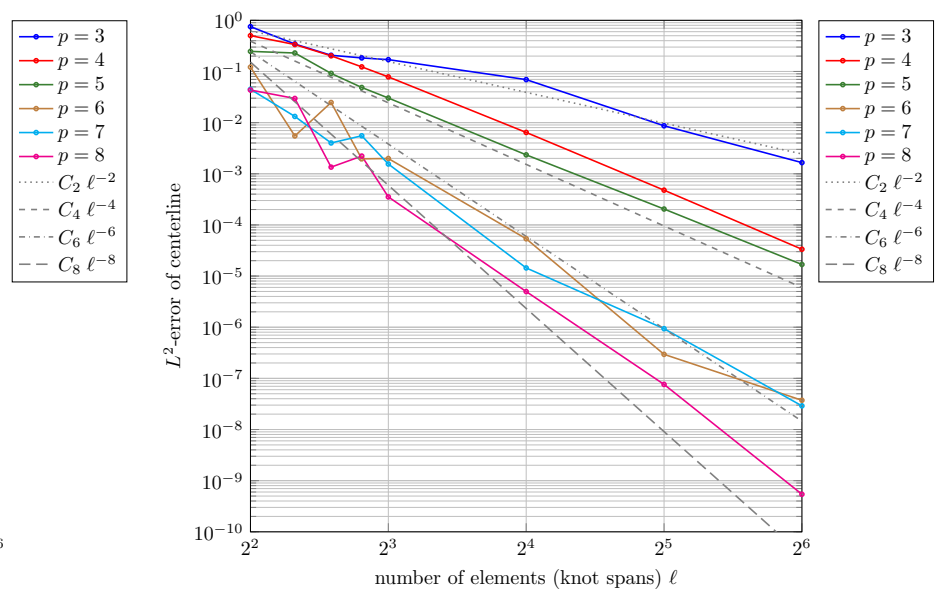

(b) $E(s)$ of degree 3 with 1 element
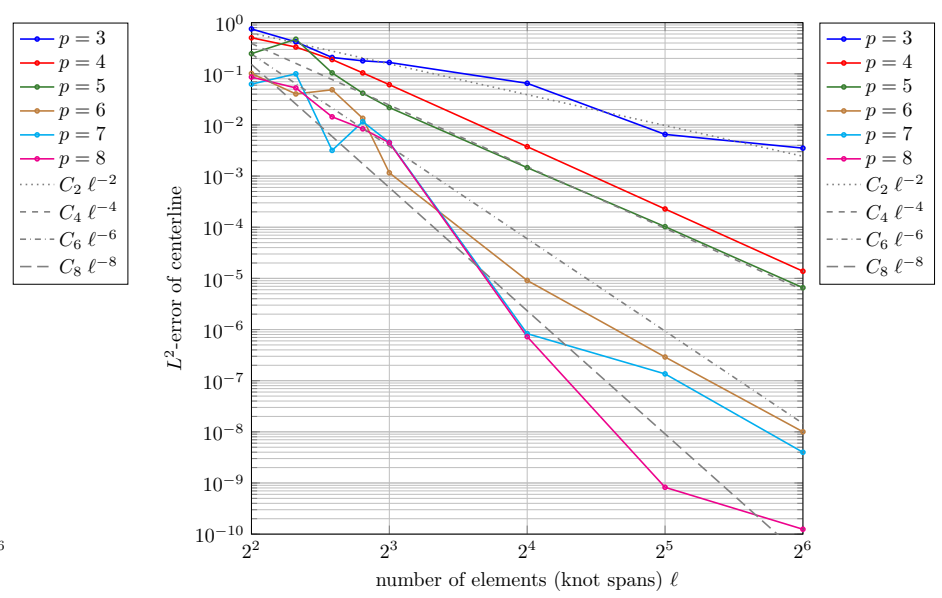

(d) $E(s)$ interpolating sinusoidal curve

Figure 6: Convergence study for axially varying Young's modulus distribution. Convergence plots show $L^{2}$-error in deformed centerline position $e_{h}=\left\|\mathbf{r}_{h}-\mathbf{r}_{*}\right\|$ for all four cases (a)-(d) of Young's modulus parameterizations

obtained. This behavior is reasonable, since the local material parameterization is collocated at suitable points, but there is an additional error component stemming from the interpolation of the sinusoidal curve.

Altogether, we can conclude from these convergence studies that the isogeometric collocation method still provides an accurate and efficient discretization method when AV cross-section parameters are used. Most importantly, the most convincing feature of collocation is preserved, i.e., high accuracy can be obtained for a low number of degrees of freedom when a higher order discretization with a relative small number of elements is employed.

Remark 1. Here, we have used a beam with a fairly large thickness-to-length ratio, i.e., $2 r / L=0.1$, since we wanted to avoid that shear locking affects the results. As mentioned above in Sect. 5. mixed methods can be used to eradicate the effects of shear locking and improve convergence rates for odd spline basis function degrees and general accuracy, i.e., error constants.

\subsubsection{Slender rods with transversally varying materials}

The use of functionally graded cross-sections with transversally varying materials does in general not affect the accuracy and convergence behavior of the discretization method, since the constitution of the cross-section only defines the constitutive parameters, see Sect. 4.1. However, when the Cosserat rod is 


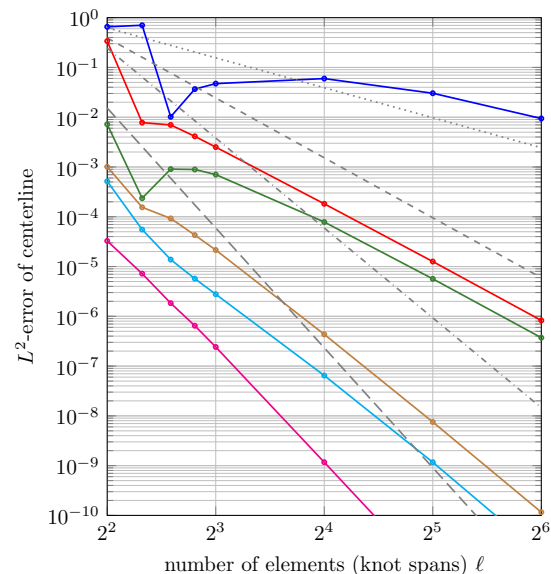

(a1) $R=0.05 \mathrm{~m}$, primal

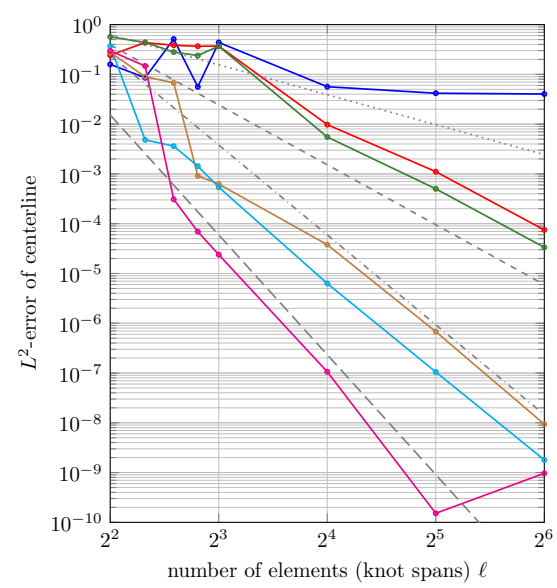

(b1) $R=0.005 \mathrm{~m}$, primal

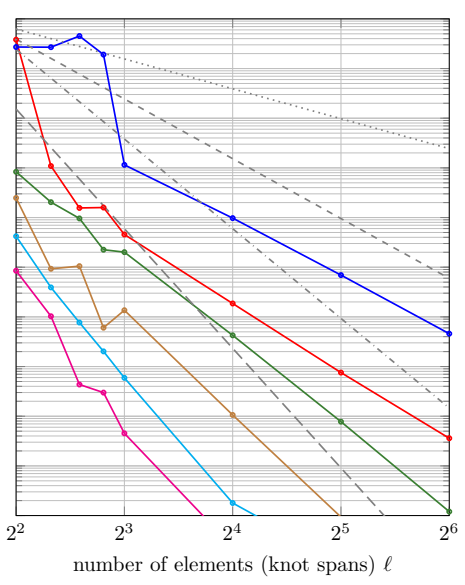

(a2) $R=0.05 \mathrm{~m}$, mixed

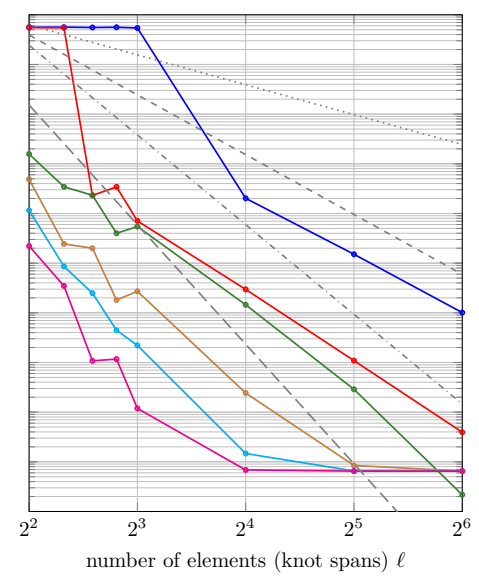

(b2) $R=0.005 \mathrm{~m}$, mixed

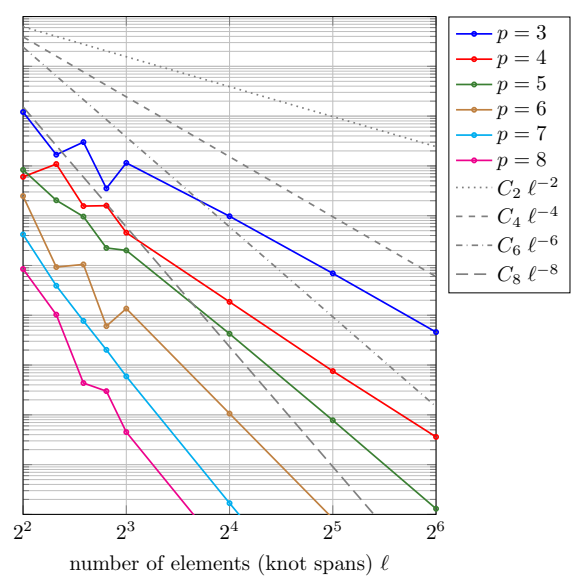

(a3) $R=0.05 \mathrm{~m}$, enhanced mixed

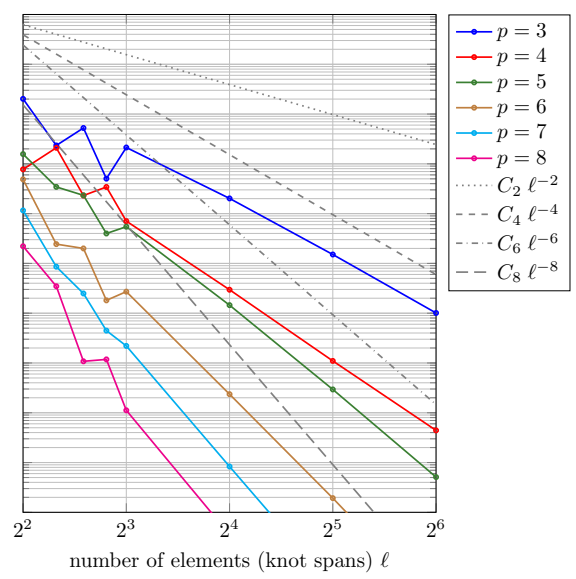

(b3) $R=0.005 \mathrm{~m}$, enhanced mixed

Figure 7: Convergence study for slender rods with transversally varying materials. Convergence plots show error in centerline position for (a) $R=0.05 \mathrm{~m}$ and (b) $R=0.005 \mathrm{~m}$ using (1) primal $(\mathbf{r}, \mathbf{q})_{h},(2)$ mixed $(\mathbf{r}, \mathbf{q}, \mathbf{n}, \mathbf{m})_{h}$, and $(3)$ enhanced mixed $\left(\mathbf{r}, \mathbf{q}, \mathbf{n}^{\varepsilon}, \mathbf{n}^{\kappa}, \mathbf{m}^{\varepsilon}, \mathbf{m}^{\kappa}\right)_{h}$ methods

slender, a mixed method should or has to be used to resolve the shear locking problem. Having introduced an extension of the mixed collocation method in Sect. 5. we now evaluate its performance for the analysis of slender rods with transversally varying material compositions.

Again, we take a rod of length $L=1.0 \mathrm{~m}$, which now has a circular, bilayer laminate cross-section, see Sect. 4.1.1. The material parameters are chosen as Young's moduli $E_{0}=100 \mathrm{MPa}, E_{1}=10 \mathrm{MPa}$ and Poisson's ratios $\nu_{0}=\nu_{1}=0.45$. Now, the radius of the cross-section is varied using (a) $R=0.05 \mathrm{~m}$ and (b) $R=0.005 \mathrm{~m}$, while the relative layer ratios are kept constant using layer heights $h_{L}=-0.5 R$. Like before, the rods are clamped at one end and moments $\hat{m}(L)=0.5 \pi C_{11}$ (which depend on $R$, see $(20)$ ) are applied at the other end incrementally over 8 load steps, deforming each rod to almost a semicircle.

For each choice of $R$, we use the conventional formulation, where only centerline positions and quaternions are discretized (primal $(\mathbf{r}, \mathbf{q})_{h}$, see Sect. 3.2), the original mixed method from [42, where additionally the translational and rotational stresses are discretized $\left(\operatorname{mixed}(\mathbf{r}, \mathbf{q}, \mathbf{n}, \mathbf{m})_{h}\right.$, see Sect. 5. Eqn. (32)), and the newly presented enhanced mixed method, where the translational and rotational contributions to the translational and rotational stresses are separately discretized (enhanced mixed $\left(\mathbf{r}, \mathbf{q}, \mathbf{n}^{\varepsilon}, \mathbf{n}^{\kappa}, \mathbf{m}^{\varepsilon}, \mathbf{m}^{\kappa}\right)_{h}, \operatorname{see}$ Sect.5. Eqn. (35) ) to compute the displaced configurations. We evaluate the $L^{2}$-errors of deformed centerline positions against reference solutions with highly refined isogeometric discretizations $(p=10, \ell=128)$ and 


\begin{tabular}{|c|c|c|c|c|c|c|c|c|c|c|}
\hline & \multicolumn{3}{|c|}{ per iteration $[\mathrm{ms}]$} & \multicolumn{3}{|c|}{$r=0.05[\mathrm{~s}]$} & \multicolumn{3}{|c|}{$r=0.005[\mathrm{~s}]$} \\
\hline & & $\mathrm{PM}$ & MM & $\mathrm{EM}$ & PM & MM & EM & PM & MM & EM \\
\hline \multirow{5}{*}{$\begin{array}{l}p=4 \\
\ell=16 \\
n=20\end{array}$} & \# load steps & - & - & - & 8 & 18 & 8 & 8 & 129 & 8 \\
\hline & & - & - & - & 64 & 246 & 46 & 106 & 2014 & 48 \\
\hline & assembly & 10.2 & 4.2 & 7.0 & 0.680 & 1.037 & 0.327 & 1.029 & 8.352 & 0.330 \\
\hline & lve & 1.5 & 2.4 & 4.8 & 0.099 & 0.622 & 0.230 & 0.155 & 4.770 & 0.225 \\
\hline & total & 11.7 & 6.6 & 11.8 & 0.779 & 1.659 & 0.557 & 1.183 & 13.121 & 0.555 \\
\hline \multirow{5}{*}{$\begin{array}{c}p=4 \\
\ell=64 \\
n=68\end{array}$} & \# loas & - & - & - & 8 & 17 & 8 & 8 & 128 & 8 \\
\hline & & - & - & - & 64 & 214 & 47 & 200 & 1774 & 48 \\
\hline & assembl & 37.9 & 24.2 & 47.2 & 2.498 & 5.275 & 2.261 & 7.353 & 42.308 & 2.222 \\
\hline & linear solve & 4.2 & 6.2 & 11.6 & 0.266 & 1.327 & 0.542 & 0.851 & 11.017 & 0.557 \\
\hline & total & 42.1 & 30.5 & 58.8 & 2.764 & 6.602 & 2.803 & 8.204 & 53.325 & 2.778 \\
\hline
\end{tabular}

Table 1: CPU times for primal (PM), mixed (MM) and enhanced mixed (EM) methods for slender rods with transversally varying materials. Assembly, linear solution and total time are shown for one iteration (in milliseconds), as well as for solving the beam displacement in (at least) 8 load steps for $r=0.05$ and $r=0.005$ (in seconds)

resulting convergence plots are shown in Fig. 7

When the rod becomes more slender, the primal method loses accuracy due to shear locking, see Fig.7a1 and Fig. 7b1. Furthermore, the convergence behavior of the Newton iteration is affected and a lot of iterations per load step are required (around 10-20), or the 8 load steps had to be further subdivided to achieve convergence for $R=0.005 \mathrm{~m}$.

The original mixed method, which is shown in Fig. 7a2 and Fig. 7b2, seems to attain high accuracy and good convergence rates for both cross-section radii. However, to obtain these results, we had to further subdivide the application of the moment from 8 to 32 and 160 (!) load steps for $R=0.05 \mathrm{~m}$ and $R=0.005 \mathrm{~m}$, respectively. Otherwise, the method is numerically unstable and the Newton iterations would not converge at all. Thus, employing this method for TV cross-sections means a tremendous increase in computational cost and is not advisable.

For the newly proposed enhanced mixed method, it can be seen in Fig. 7a3 and Fig. 7b3 that almost the same high accuracy and ideal convergence rates can be achieved independently of the slenderness of the cross-section. Furthermore, the method only needed very few Newton iterations for each of the 8 load steps, typically only 6 iterations for a relative accuracy of $10^{-9}$.

In Table 1 we have summarized the CPU times required for assembly and solution for the three methods with $p=4$ and $\ell=16,64$, using an Intel ${ }^{\circledR}$ Core $^{\mathrm{TM}}$ i7-5960X $3.00 \mathrm{GHz}$ PC with 32 GB RAM in single-core mode and UMFPACK direct sparse linear solver 62. Per iteration, the mixed method (MM) is faster than the primal (PM) and enhanced mixed method (EM), but while PM and MM suffer from locking and instability, and thus need a lot of load steps and iterations, EM is very efficient and overall much faster than both PM and MM - and at the same time also more accurate than PM.

Thus, we can conclude that the newly developed enhanced mixed method overcomes the shear locking problem and provides a stable and efficient solution process for slender rods with TV cross-sections.

\subsection{Applications of rods with spatially varying materials}

Next, we study a few simple applications of modeling of 3D beams with spatially varying materials, i.e., beams with both axially and transversally varying cross-section parameters, including rotated cross-section orientations.

\subsubsection{Homogeneous vs. graded vs. bilayer cross-sections}

Functionally graded, TV cross-sections with asymmetric Young's modulus distributions, such as the bilayer laminates and continuously graded variations introduced in Sect. 4.1, are characterized by the coupling of tension and bending deformations. This enables the design of rods with very specific and tailored deformation behavior. 
(a)

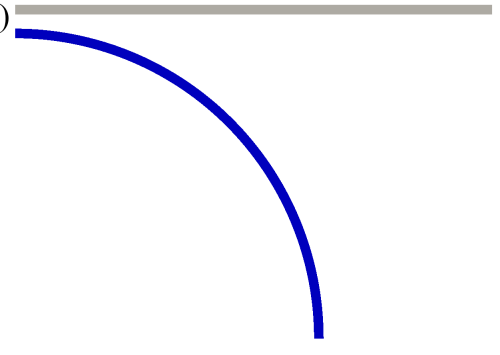

(b)

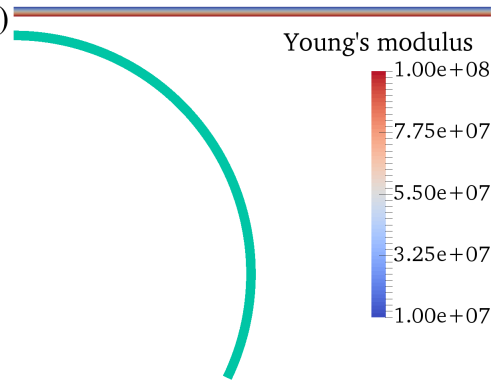

(c)

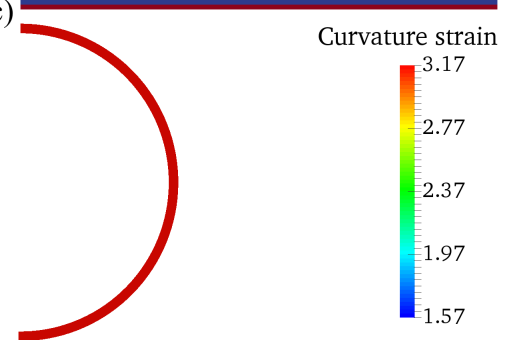

Figure 8: Homogeneous vs. graded vs. bilayer cross-sections. Initial rods with (a) homogeneous cross-section, (b) linear transverse grading and (c) bilayer laminate (on top, colored by Young's modulus distribution) and deformed, curved configurations after moment was applied (below, colored by curvature strains)

To demonstrate this, we compare the bending behavior of three slender cantilever beams of length $L=1$ $\mathrm{m}$ with rectangular cross-sections with $b=3 \mathrm{~mm}, h=2 \mathrm{~mm}, E_{0}=100 \mathrm{MPa}, E_{1}=10 \mathrm{MPa}$, and $\nu=0.45$ with (a) homogeneous cross-section with $E=0.5\left(E_{0}+E_{1}\right)$, (b) continuous, linear grading with $p=1$, and (c) bilayer laminate with $h_{L}=0 \mathrm{~mm}$. Consequently, all respective coefficients of the constitutive matrices $\mathbf{A}$ and $\mathbf{C}$ are equal for the three cases, but $B_{31}^{(a)}=0$ and $B_{31}^{(c)}=\frac{3}{2} B_{31}^{(b)}$.

We apply a moment $\hat{m}(L)=0.5 \pi C_{11}$ at the free end of the beams and discretize them using the enhanced mixed method with $p=6, \ell=16$ to compute the deformations. The initial beams and their deformed configurations for all cases are shown in Fig. 8. Application of a moment results in constant extensional strains and bending curvatures, which are:

$$
\begin{array}{lll}
\varepsilon_{3}^{(a)}=0, & \varepsilon_{3}^{(b)}=0.0055, & \varepsilon_{3}^{(c)}=0.0129, \\
\kappa_{1}^{(a)}=1.571, & \kappa_{1}^{(b)}=2.022, & \kappa_{1}^{(c)}=3.155 .
\end{array}
$$

While the homogeneous beam bends by exactly $90^{\circ}$, the linearly graded beam bends about $115^{\circ}$, and the bilayer beam bends even slightly more than $180^{\circ}$. This provides an interesting insight into the behavior and resulting design opportunities of beams with TV material compositions.

Remark 2. In this simple case of 2-dimensional beam bending, the above-computed quantities can also be determined analytically through the relationship:

$$
\left(\begin{array}{ll}
A_{33} & B_{31} \\
B_{31} & C_{11}
\end{array}\right)\left(\begin{array}{l}
\varepsilon_{3} \\
\kappa_{1}
\end{array}\right)=\left(\begin{array}{c}
0 \\
\hat{m}
\end{array}\right)
$$

\subsubsection{Bilayer cross-section with axially varying geometric parameters and rotation}

Now, we introduce an application where a TV cross-section, here a bilayer laminate, has AV geometric properties, here cross-section radius, layer height and rotation, see Fig. 9 .

The bilayer cantilever beam has length $L=1 \mathrm{~m}$, radius parameter $r_{0}=0.025 \mathrm{~m}$, Young's moduli $E_{0}=100 \mathrm{MPa}$ and $E_{1}=10 \mathrm{MPa}$, and $\nu=0.45$. The following cross-section properties are axially varying, here parameterized linearly from $s=0$ to $s=1$ : radius $r(s)=r_{0}(2-s)$, layer height ratio $h_{R}(s)=0.25+0.5 s$, and rotation angle $\alpha(s)=\pi s$. Thus, we have $p^{u}=1, \ell^{u}=1, n^{u}=2$ and the parameter vector is $\mathbf{u} \equiv\left(r, h_{R}, \alpha\right)^{\top}$. A follower moment $\hat{\mathbf{m}}(L)=\mathbf{R}^{\top}\left(0.5 \pi C_{22}(L / 2), 0,0\right)^{\top}$ is applied on its free end.

The initial shape and material constitution of the beam and its deformed configuration are visualized in Fig. 9 a-b. As can be seen from the deformed shapes and the values of curvature strains $\kappa_{1}$ and $\kappa_{2}$, the beam undergoes a highly complex and nonlinear bending behavior with coupling of both curvature modes, twist and tension. Our isogeometric modeling and analysis framework enables convenient modeling of such complex beams and their accurate analysis, here using the newly introduced enhanced mixed method, as can be seen from the convergence plot for the $L^{2}$-error of displacements shown in Fig. 9 . 
(a)

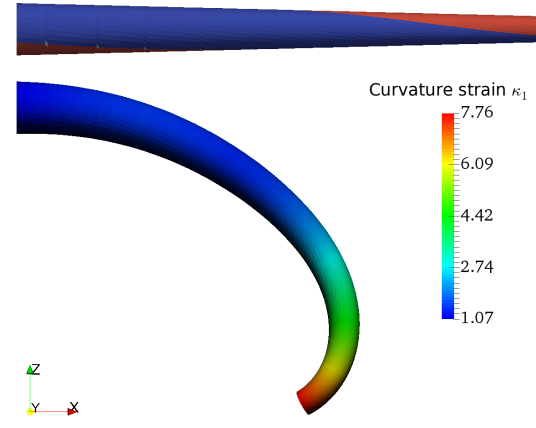

(b)

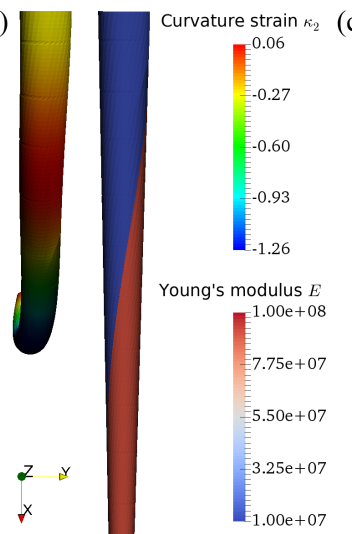

(c)

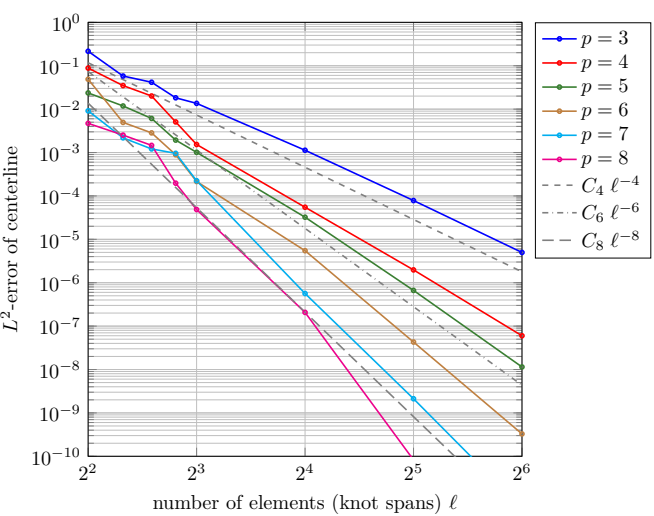

Figure 9: Bilayer cross-section with axially varying geometric parameters and rotation. Initial (colored by Young's modulus) and deformed (colored by curvature strains) rod configurations in (a) $x / z$-view and (b) $y /-x$-view. (c) Convergence of $L^{2}$-error of displacements.

\subsection{Application to a graded $3 D$ lattice structure}

Finally, we apply the concept of AV and TV material compositions to a graded, lattice-like rod structure. This application points towards the design of structures with spatially varying materials for specific deformation behavior, instabilities, or energy absorption capabilities, such as the soft, compliant lattice structures presented in 63.

Here, we introduce a tire-like, cylindrical 3D lattice structure with outer radius $100 \mathrm{~mm}$, inner radius 70 $\mathrm{mm}$, and height $30 \mathrm{~mm}$, see Fig. 10. We use a honeycomb-type lattice unit cell structure with 16 cells in circumferential direction of the cylinder. The individual struts have an axially varying radius, which varies in radial direction of the cylindrical geometry from $1.5 \mathrm{~mm}$ at the inner side to $1.0 \mathrm{~mm}$ at the outer side. Struts at the inner and outer sides of the cylindrical geometry are transversally varying bilayers with height ratio $h_{R}=0.8$, where the smaller layer has Young's modulus $E_{1}=1.2 \mathrm{GPa}$ and the larger layer and all uniform rods have Young's modulus $E_{0}=0.6 \mathrm{MPa}$. Thus, the small layers act like a very stiff coating on the otherwise rather soft structure. The Poisson's ratios are $\nu_{0}=\nu_{1}=0.45$. These material parameters correspond to typical polymer inkjet 3D printing materials, as for instance used in 63.

For axially varying cross-section properties, here the radius, we simply use a linear global parameterization, i.e., $\mathbf{u} \equiv r$ and $p_{u}=1, \ell_{u}=1$. The isogeometric discretization of the totally 512 rods is done using the mixed methods with $p=6, \ell=8$. The original mixed method is used for interior rods with homogeneous cross-sections and the enhanced mixed method is used for the bilayers, since there is a strong difference of Young's moduli of more than three orders of magnitude.

For the mechanical simulation of the structure, the rods at the right end are clamped and a displacement of $40 \mathrm{~mm}$ in $x$-direction is incrementally applied to the rods on the left end over 30 load steps. This results in an overall compression of $20 \%$ of the structure in $x$-direction and to complex deformation patterns of the individual rods, including torsion and double-curved bending deformations, see Fig. 10.

\section{Summary and conclusions}

We have introduced a fully isogeometric modeling and analysis method for 3D beams with spatially varying material and geometric parameters. The beams were modeled using the geometrically exact, nonlinear Cosserat rod theory and discretized by an isogeometric collocation method. Axially varying material and geometric properties of the beam cross-sections are also parameterized using spline functions, allowing a convenient and flexible implementation of the method in the IGA context. Effects of the axially varying parameterizations on convergence behavior were investigated, showing that there are no significant implications on convergence rates of the collocation method. Transversally varying, functionally graded 


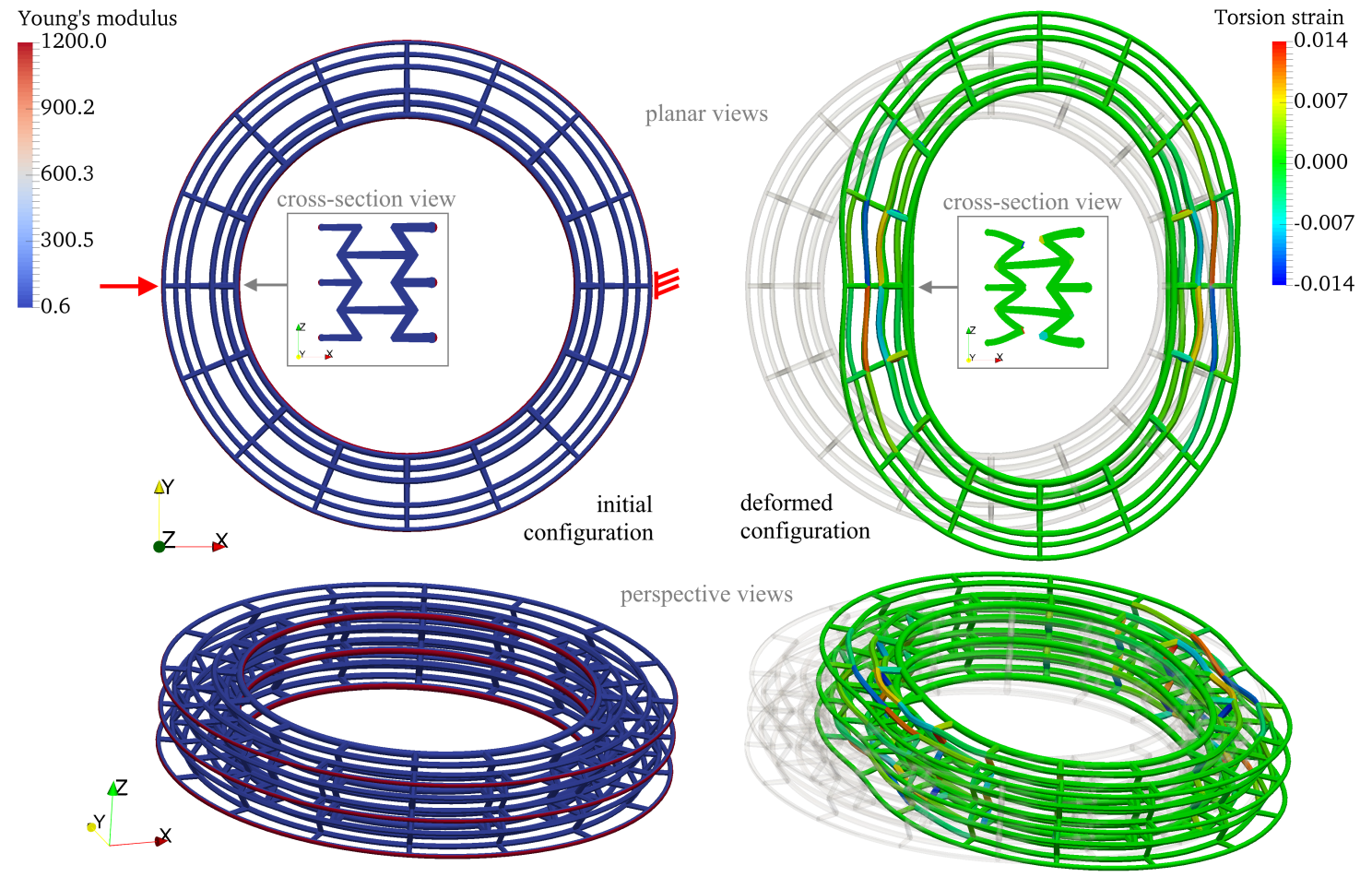

Figure 10: Graded 3D lattice structure. The initial, tire-like lattice structure is shown on the left side, colored by the Young's modulus distribution. The deformed structure after application of $40 \mathrm{~mm}$ deformation is shown on the right side, colored by the torsion strain $\kappa_{3}$

cross-sections were also implemented as bilayer laminates and with continuous grading of Young's modulus. To overcome shear locking and convergence issues for those transversally varying beams, a new enhanced mixed collocation method was introduced and successfully numerically evaluated.

The next step based on this work is the implementation of isogeometric design optimization methods, that optimize the spatially varying material and geometric parameters of the cross-sections. Furthermore, shape and (ground-structure) topology optimization could be used to implement an even more flexible optimization framework for 3-dimensional beam structures. Potential applications for these methods, which would already be realizable with current advanced manufacturing technologies, include design and optimization of soft, multi-material and composite rod structures that are subject to large deformations and instabilities, such as soft robotic actuators, artificial muscles and flexible space structures.

To overcome the limitations of the Cosserat rod model in terms of the approximation of shear stress distribution for axially-varying beams, a model could be derived that enables the coupling of all forces and moments with stress and strain components. This model could then be validated against the current model, a beam model where a continuum hyperelastic constitutive law is used and integrated over the cross-section, see [29], and full 3D finite element analysis.

\section{Acknowledgements}

The authors acknowledge support from the SUTD Digital Manufacturing and Design (DManD) Centre, supported by the Singapore National Research Foundation. This research project is partially funded by the SUTD SRG and an internal grant from HKUST (R9429). Furthermore, we would like to thank Sang-In Park from SUTD for help with providing validation results. 


\section{A. Appendix}

\section{A.1. Constitutive coefficients for bilayer laminate cross-sections}

We continue the derivation and calculation of the constitutive coefficients for bilayer laminate crosssections as presented in Sect. 4.1.1.

For the rectangular cross-section with width $b$ and height $h$, the integrals in 20 can be analytically evaluated as:

$$
\begin{array}{ll}
\bar{A}_{33}^{0}=\int_{-b / 2}^{b / 2} \int_{-h / 2}^{h_{L}} d x_{2} d x_{1}=b\left(\frac{h}{2}+h_{L}\right), & \bar{A}_{33}^{1}=\int_{-b / 2}^{b / 2} \int_{h_{L}}^{h / 2} d x_{2} d x_{1}=b\left(\frac{h}{2}-h_{L}\right), \\
\bar{B}_{31}^{0}=\int_{-b / 2}^{b / 2} \int_{-h / 2}^{h_{L}} x_{2} d x_{2} d x_{1}=\frac{b}{2}\left(h_{L}^{2}-\frac{h^{2}}{4}\right), & \bar{B}_{31}^{1}=\int_{-b / 2}^{b / 2} \int_{h_{L}}^{h / 2} x_{2} d x_{2} d x_{1}=\frac{b}{2}\left(\frac{h^{2}}{4}-h_{L}^{2}\right), \\
\bar{C}_{11}^{0}=\int_{-b / 2}^{b / 2} \int_{-h / 2}^{h_{L}} x_{2}^{2} d x_{2} d x_{1}=\frac{b}{3}\left(\frac{h^{3}}{8}+h_{L}^{3}\right), & \bar{C}_{11}^{1}=\int_{-b / 2}^{b / 2} \int_{h_{L}}^{h / 2} x_{2}^{2} d x_{2} d x_{1}=\frac{b}{3}\left(\frac{h^{3}}{8}-h_{L}^{3}\right), \\
\bar{C}_{22}^{0}=\int_{-b / 2}^{b / 2} \int_{-h / 2}^{h_{L}} x_{1}^{2} d x_{2} d x_{1}=\frac{b^{3}}{12}\left(\frac{h}{2}+h_{L}\right), & \bar{C}_{22}^{1}=\int_{-b / 2}^{b / 2} \int_{h_{L}}^{h / 2} x_{1}^{2} d x_{2} d x_{1}=\frac{b^{3}}{12}\left(\frac{h}{2}-h_{L}\right) .
\end{array}
$$

For the circular cross-section with radius $r$, the analytical evaluation of the integrals in $(20)$ is a little more complex. Defining the layer angle $\theta=2 \arccos \left(h_{L} / r\right)$ and layer width $b_{L}=r \sin (\theta / 2)$ in addition to the layer height $h_{L}$, and parameterizing the boundaries of the integrals either as $x_{1}\left(x_{2}\right)=r \sin \left(\arccos \left(x_{2} / r\right)\right)$ or $x_{2}\left(x_{1}\right)=r \cos \left(\arcsin \left(x_{1} / r\right)\right)$, it follows:

$$
\begin{aligned}
& \bar{A}_{33}^{1}=\int_{-b_{L}}^{b_{L}} \int_{h_{L}}^{x_{2}\left(x_{1}\right)} d x_{2} d x_{1}=\int_{-b_{L}}^{b_{L}} r \cos \left(\arcsin \left(x_{1} / r\right)\right)-h_{L} d x_{1}=\frac{r^{2}}{2}(\theta-\sin \theta), \\
& \bar{A}_{33}^{0}=\pi r^{2}-\bar{A}_{33}^{1}=\frac{r^{2}}{2}(2 \pi-\theta+\sin \theta), \\
& \bar{B}_{31}^{1}=\int_{-b_{L}}^{b_{L}} \int_{h_{L}}^{x_{2}\left(x_{1}\right)} x_{2} d x_{2} d x_{1}=\frac{1}{2} \int_{-b_{L}}^{b_{L}} r^{2} \cos ^{2}\left(\arcsin \left(x_{1} / r\right)\right)-h_{L}^{2} d x_{1}=\frac{2}{3} r^{3} \sin ^{3}(\theta / 2), \\
& \bar{B}_{31}^{0}=-\bar{B}_{31}^{1}=-\frac{2}{3} r^{3} \sin ^{3}(\theta / 2), \\
& \bar{C}_{11}^{1}=\int_{-b_{L}}^{b_{L}} \int_{h_{L}}^{x_{2}\left(x_{1}\right)} x_{2}^{2} d x_{2} d x_{1}=\frac{1}{3} \int_{-b_{L}}^{b_{L}} r^{3} \cos ^{3}\left(\arcsin \left(x_{1} / r\right)\right)-h_{L}^{3} d x_{1}=\frac{r^{4}}{16}(2 \theta-\sin (2 \theta)), \\
& \bar{C}_{11}^{0}=\frac{1}{4} \pi r^{4}-\bar{C}_{11}^{1}=\frac{r^{4}}{16}(4 \pi-2 \theta+\sin (2 \theta)), \\
& \bar{C}_{22}^{1}=\int_{-b_{L}}^{b_{L}} \int_{h_{L}}^{x_{2}\left(x_{1}\right)} x_{1}^{2} d x_{2} d x_{1}=\int_{-b_{L}}^{b_{L}} x_{1}^{2}\left(r \cos \left(\arcsin \left(x_{1} / r\right)\right)-h_{L}\right) d x_{1}=\frac{r^{4}}{48}(6 \theta-8 \sin (\theta)+\sin (2 \theta)), \\
& \bar{C}_{22}^{0}=\frac{1}{4} \pi r^{4}-\bar{C}_{22}^{1}=\frac{r^{4}}{48}(12 \pi-6 \theta+8 \sin (\theta)-\sin (2 \theta)) .
\end{aligned}
$$

For both cases, rectangular and circular cross-sections, the torsion constant $C_{33}$ cannot be determined as a closed-form solution in terms of the parameters $h_{L}, b$ and $h$ resp. $r$, and $E_{0}, E_{1}$, and thus have to be determined through solving a 2-dimensional PDE problem on the cross-section domain $S$, see [25]. For circular cross-sections, we have obtained an approximation from numerical data as $C_{33}=E_{0} \bar{C}_{33}^{0}+E_{1} \bar{C}_{33}^{1}$ with:

$$
\begin{aligned}
& \bar{C}_{33}^{0}=\frac{1}{16} \pi r^{4}\left(2\left(1+h_{L} / r\right)^{2}+\left(1+h_{L} / r\right)^{3}\right), \\
& \bar{C}_{33}^{1}=\frac{1}{16} \pi r^{4}\left(2\left(1-h_{L} / r\right)^{2}+\left(1-h_{L} / r\right)^{3}\right),
\end{aligned}
$$

which was then used in the numerical applications presented. 


\section{A.2. Constitutive coefficients for continuously graded cross-sections}

We continue the derivation and calculation of the constitutive coefficients for continuously graded, rectangular cross-sections as presented in Sect. 4.1.2. The parameters of the cross-section are width $b$, height $h$ and Young's modulus distribution $E\left(x_{2}\right)=E_{0}+\left(E_{1}-E_{0}\right)\left(\frac{1}{2}+\frac{x_{2}}{h}\right)^{p}$ with $E_{0}, E_{1}, p>0$. Then, the analytical evaluation of the integrals yields:

$$
\begin{aligned}
A_{33} & =\int_{-b / 2}^{b / 2} \int_{-h / 2}^{h / 2} E\left(x_{2}\right) d x_{2} d x_{1}=b h \frac{p E_{0}+E_{1}}{p+1} \\
B_{31} & =\int_{-b / 2}^{b / 2} \int_{-h / 2}^{h / 2} x_{2} E\left(x_{2}\right) d x_{2} d x_{1}=\frac{b h^{2}}{2} \frac{p\left(E_{1}-E_{0}\right)}{(p+1)(p+2)} \\
C_{11} & =\int_{-b / 2}^{b / 2} \int_{-h / 2}^{h / 2} x_{2}^{2} E\left(x_{2}\right) d x_{2} d x_{1}=\frac{b h^{3}}{12} \frac{p E_{0}\left(8+3 p+p^{2}\right)+3 E_{1}\left(2+p+p^{2}\right)}{(p+1)(p+2)(p+3)} \\
C_{22} & =\int_{-b / 2}^{b / 2} \int_{-h / 2}^{h / 2} x_{1}^{2} E\left(x_{2}\right) d x_{2} d x_{1}=\frac{b^{3} h}{12} \frac{p E_{0}+E_{1}}{p+1} \\
C_{33} & \equiv C_{33}\left(b, h, E_{0}, E_{1}, p\right) .
\end{aligned}
$$

\section{Bibliography}

[1] I. Gibson, D. Rosen, and B. Stucker. Additive Manufacturing Technologies: 3D Printing, Rapid Prototyping, and Direct Digital Manufacturing. Springer-Verlag New York, second edition, 2015.

[2] B.G. Compton and J.A. Lewis. 3D-printing of lightweight cellular composites. Advanced Materials, 26(34):5930-5935, 2014.

[3] X. Zheng, H. Lee, T.H. Weisgraber, M. Shusteff, J. DeOtte, E.B. Duoss, J.D. Kuntz, M.M. Biener, Q. Ge, J.A. Jackson, S.O. Kucheyev, N.X. Fang, and C.M. Spadaccini. Ultralight, ultrastiff mechanical metamaterials. Science, 344(6190):13731377, 2014.

[4] A.P. Garland and G. Fadel. Design and manufacturing functionally gradient material objects with an off the shelf 3D printer: Challenges and solutions. ASME Journal of Mechanical Design, 137(11):111407, 2015.

[5] A.D.B.L. Ferrera, P.R.O. Nóvoa, and A.T. Marques. Multifunctional material systems: A state-of-the-art review. Composite Structures, 151:3-35, 2016.

[6] O. Weeger, Y.S.B. Kang, S.-K. Yeung, and M.L. Dunn. Optimal design and manufacture of active rod structures with spatially variable materials. 3D Printing and Additive Manufacturing, 3(4):204-215, 2016.

[7] N. Hu and R. Burgueõ. Buckling-induced smart applications: Recent advances and trends. Smart Materials and Structures, 24(6):063001, 2015.

[8] Q. Ge, A.H. Sakhaei, H. Lee, C.K. Dunn, N.X. Fang, and M.L. Dunn. Multimaterial 4D printing with tailorable shape memory polymers. Scientific Reports, 6:31110, 2016.

[9] Z. Ding, C. Yuan, X. Peng, T. Wang, H.J. Qi, and M.L. Dunn. Direct 4D printing via active composite materials. Science Advances, 3(4):e1602890, 2017.

[10] Z. Ding, O. Weeger, H.J. Qi, and M.L. Dunn. 4D rods: 3D structures via programmable 1D composite rods. Materials \& Design, 137:256-265, 2018.

[11] T.W. Murphey. Large strain composite materials in deployable space structures. In 17th International Conference on Composite Materials, Edinburgh, United Kingdom, 2009. The British Composites Society.

[12] T.W. Murphey, D. Turse, and L. Adams. TRAC boom structural mechanics. In 4th AIAA Spacecraft Structures Conference, Grapevine, TX, United States, 2017. American Institute of Aeronautics and Astronautics.

[13] S. Suresh and A. Mortensen. Fundamentals of functionally graded materials. IOM Communications Limited, 1998.

[14] D.H. Hodges. Nonlinear Composite Beam Theory, volume 213 of Progress in Astronautics and Aeronautics. American Institute of Aeronautics and Astronautics, 2006.

[15] B.V. Sankar. An elasticity solution for functionally graded beams. Composites Science and Technology, 61(5):689-696, 2001.

[16] Z. Zhong and T. Yu. Analytical solution of a cantilever functionally graded beam. Composites Science and Technology, $67(3-4): 481-488,2007$.

[17] X.-F. Li. A unified approach for analyzing static and dynamic behaviors of functionally graded Timoshenko and EulerBernoulli beams. Journal of Sound and Vibration, 318(4-5):1210-1229, 2008.

[18] A. Chakraborty, S. Gopalakrishnan, and J.N. Reddy. A new beam finite element for the analysis of functionally graded materials. International Journal of Mechanical Sciences, 45:519-539, 2003.

[19] S.S. Antman. Nonlinear Problems of Elasticity, volume 107 of Applied Mathematical Sciences. Springer New York, 2005.

[20] S. Eugster. Geometric Continuum Mechanics and Induced Beam Theories, volume 75 of Lecture Notes in Applied and Computational Mechanics. Springer International Publishing, 2015. 
[21] E. Reissner. On finite deformations of space-curved beams. Zeitschrift für angewandte Mathematik und Physik ZAMP, 32(6):734-744, 1981.

[22] J.C. Simo. A finite strain beam formulation. The three-dimensional dynamic problem. Part I. Computer Methods in Applied Mechanics and Engineering, 49(1):55-70, 1985.

[23] L. Vu-Quoc, H. Deng, and I.K. Ebcioğlu. Multilayer beams: A geometrically exact formulation. Journal of Nonlinear Science, 6(3):239-270, 1996

[24] U. Eroglu. Large deflection analysis of planar curved beams made of functionally graded materials using variational iterational method. Composite Structures, 136:204-216, 2016.

[25] M. Bîrsan, H. Altenbach, T. Sadowski, V.A. Eremeyev, and D. Pietras. Deformation analysis of functionally graded beams by the direct approach. Composites Part B: Engineering, 43(3):1315-1328, 2012.

[26] M. Bîrsan, T. Sadowski, L. Marsavina, E. Linul, and D. Pietras. Mechanical behavior of sandwich composite beams made of foams and functionally graded materials. International Journal of Solids and Structures, 50:519-530, 2013.

[27] L.-L. Ke, J. Yang, and S. Kitipornchai. Nonlinear free vibration of functionally graded carbon nanotube-reinforced composite beams. Composite Structures, 92(3):676-683, 2010.

[28] W. Yu, D.H. Hodges, V. Volovoi, and C.E.S. Cesnik. On Timoshenko-like modeling of initially curved and twisted composite beams. International Journal of Solids and Structures, 39(19):5101-5121, 2002.

[29] S. Klinkel and S. Govindjee. Using finite strain 3D-material models in beam and shell elements. Engineering Computations, 19(8):902-921, 2002.

[30] S. Timoshenko and J.N. Goodier. Theory of Elasticity. McGraw-Hill Book Company, Inc., 2nd edition edition, 1951.

[31] O.T. Bruhns. Advanced Mechanics of Solids. Springer-Verlag Berlin Heidelberg GmbH, 2003.

[32] J. Murín and V. Kutiš. 3D-beam element with continuous variation of the cross-sectional area. Computers and Structures, 80:329-338, 2002.

[33] J. Murín and V. Kutiš. Geometrically non-linear truss element with varying stiffness. Engineering Mechanics, 13(6):435$452,2006$.

[34] V. Kutiš, J. Murín, R. Belák, and J. Paulech. Beam element with spatial variation of material properties for multiphysics analysis of functionally graded materials. Computers and Structures, 89:1192-1205, 2011.

[35] G. Balduzzi, M. Aminbaghai, E. Sacco, J. Füssl, J. Eberhardsteiner, and F. Auricchio. Non-prismatic beams: A simple and effective Timoshenko-like model. International Journal of Solids and Structures, 90:236-250, 2016.

[36] K.-J. Bathe and S. Bolourchi. Large displacement analysis of three-dimensional beam structures. International Journal for Numerical Methods in Engineering, 14(7):961-986, 1979.

[37] J.C. Simo and L. Vu-Quoc. A three-dimensional finite-strain rod model. Part II: Computational aspects. Computer Methods in Applied Mechanics and Engineering, 58(1):79-116, 1986.

[38] A. Ibrahimbegović. On finite element implementation of geometrically nonlinear reissner's beam theory: three-dimensional curved beam elements. Computer Methods in Applied Mechanics and Engineering, 122(1):11-26, 1995.

[39] M. Bergou, M. Wardetzky, S. Robinson, B. Audoly, and E. Grinspun. Discrete elastic rods. ACM Trans. Graph., 27(3):63:1-63:12, August 2008.

[40] P. Jung, S. Leyendecker, J. Linn, and M. Ortiz. A discrete mechanics approach to the Cosserat rod theory-part 1: static equilibria. International Journal for Numerical Methods in Engineering, 85(1):31-60, 2011.

[41] E. Marino. Isogeometric collocation for three-dimensional geometrically exact shear-deformable beams. Computer Methods in Applied Mechanics and Engineering, 307:383-410, 2016.

[42] O. Weeger, S.-K. Yeung, and M.L. Dunn. Isogeometric collocation methods for Cosserat rods and rod structures. Computer Methods in Applied Mechanics and Engineering, 316:100-122, 2017.

[43] T.J.R. Hughes, J.A. Cottrell, and Y. Bazilevs. Isogeometric analysis: CAD, finite elements, NURBS, exact geometry and mesh refinement. Computer Methods in Applied Mechanics and Engineering, 194(39-41):4135-4195, 2005.

[44] J. A. Cottrell, T. J. R. Hughes, and A. Reali. Studies of refinement and continuity in isogeometric structural analysis. Computer Methods in Applied Mechanics and Engineering, 196:4160-4183, 2007.

[45] R. Bouclier, T. Elguedj, and A. Combescure. Locking free isogeometric formulations of curved thick beams. Computer Methods in Applied Mechanics and Engineering, 245-246:144-162, 2012.

[46] L. Greco and M. Cuomo. B-spline interpolation of Kirchhoff-Love space rods. Computer Methods in Applied Mechanics and Engineering, 256:251-269, 2013.

[47] O. Weeger, U. Wever, and B. Simeon. Isogeometric analysis of nonlinear Euler-Bernoulli beam vibrations. Nonlinear Dynamics, 72(4):813-835, 2013.

[48] F. Auricchio, L. Beirão da Veiga, T.J.R. Hughes, A. Reali, and G. Sangalli. Isogeometric collocation methods. Mathematical Models and Methods in Applied Sciences, 20(11):2075-2107, 2010.

[49] A. Reali and T.J.R. Hughes. An introduction to isogeometric collocation methods. In G. Beer and S. Bordas, editors, Isogeometric Methods for Numerical Simulation, volume 561 of CISM International Centre for Mechanical Sciences, pages 173-204. Springer, 2015.

[50] D. Schillinger, J. A. Evans, A. Reali, M. A. Scott, and T. J. R. Hughes. Isogeometric collocation: Cost comparison with Galerkin methods and extension to adaptive hierarchical NURBS discretizations. Computer Methods in Applied Mechanics and Engineering, 267:170-232, 2013.

[51] F. Auricchio, L. Beirão da Veiga, J. Kiendl, C. Lovadina, and A. Reali. Locking-free isogeometric collocation methods for spatial Timoshenko rods. Computer Methods in Applied Mechanics and Engineering, 263:113-126, 2013.

[52] J. Kiendl, F. Auricchio, T.J.R. Hughes, and A. Reali. Single-variable formulations and isogeometric discretizations for shear deformable beams. Computer Methods in Applied Mechanics and Engineering, 284:988-1004, 2015.

[53] F. Auricchio, L. Beirão da Veiga, J. Kiendl, C. Lovadina, and A. Reali. Isogeometric collocation mixed methods for rods. 
Discrete and Continuous Dynamical Systems - Series S, 9(1):33-42, 2016.

[54] G. Balduzzi, S. Morganti, F. Auricchio, and A. Reali. Non-prismatic Timoshenko-like beam model: Numerical solution via isogeometric collocation. Computers and Mathematics with Applications, 74, 2017.

[55] F. Auricchio, L. Beirão da Veiga, T.J.R. Hughes, A. Reali, and G. Sangalli. Isogeometric collocation for elastostatics and explicit dynamics. Computer Methods in Applied Mechanics and Engineering, 249-252:2-14, 2012. Higher Order Finite Element and Isogeometric Methods.

[56] J. Kiendl, F. Auricchio, L. Beir ao da Veiga, C. Lovadina, and A. Reali. Isogeometric collocation methods for the ReissnerMindlin plate problem. Computer Methods in Applied Mechanics and Engineering, 284:489-507, 2015. Isogeometric Analysis Special Issue.

[57] F. Maurin, F. Greco, L. Coox, D. Vandepitte, and W. Desmet. Isogeometric collocation for Kirchhoff-Love plates and shells. Computer Methods in Applied Mechanics and Engineering, 329:396-420, 2018.

[58] R. Kruse, N. Nguyen-Thanh, L. De Lorenzis, and T.J.R. Hughes. Isogeometric collocation for large deformation elasticity and frictional contact problems. Computer Methods in Applied Mechanics and Engineering, 296:73-112, 2015.

[59] J. A. Cottrell, T. J. R. Hughes, and Y. Bazilevs. Isogeometric Analysis: Toward Integration of CAD and FEA. John Wiley \& Sons, Ltd, 2009.

[60] L.A. Piegl and W. Tiller. The NURBS Book. Monographs in Visual Communication. Springer, 1997.

[61] E. Marino. Locking-free isogeometric collocation formulation for three-dimensional geometrically exact shear-deformable beams with arbitrary initial curvature. Computer Methods in Applied Mechanics and Engineering, 324:546-572, 2017.

[62] T.A. Davis. Algorithm 832: UMFPACK V4.3 - an unsymmetric-pattern multifrontal method. ACM Transactions on Mathematical Software (TOMS), 30(2):196-199, 2004

[63] O. Weeger, N. Boddeti, S.-K. Yeung, S. Kaijima, and M.L. Dunn. Digital design and nonlinear simulation for additive manufacturing of soft lattice structures. Additive Manufacturing, 2018. Under review. 